\title{
EXACT WKB ANALYSIS AND CLUSTER ALGEBRAS II: SIMPLE POLES, ORBIFOLD POINTS, AND GENERALIZED CLUSTER ALGEBRAS
}

\author{
KOHEI IWAKI AND TOMOKI NAKANISHI
}

\begin{abstract}
This is a continuation of developing mutation theory in exact WKB analysis using the framework of cluster algebras. Here we study the Schrödinger equation on a compact Riemann surface with turning points of simple-pole type. We show that the orbifold triangulations by Felikson, Shapiro, and Tumarkin provide a natural framework of describing the mutation of Stokes graphs, where simple poles correspond to orbifold points. We then show that under the mutation of Stokes graphs around simple poles the Voros symbols mutate as the variables of generalized cluster algebras introduced by Chekhov and Shapiro.
\end{abstract}

\section{INTRODUCTION}

This is a continuation of the paper [IN14, where the mutation theory in exact $W K B$ analysis using the framework of cluster algebras was initiated. The exact WKB analysis is a method to study the WKB solutions of the Schrödinger equation using the Borel resummation. It was initiated by Voros Vor83, and developed by Aoki, Kawai, and Takei AKT91, KT05, Delabaere, Dillinger, and Pham [DDP93, DP99, and others. Meanwhile, cluster algebras were introduced by Fomin and Zelevinsky [FZ03, FZ07]. They were originally introduced to study Lie theory, but nowadays they are recognized as a common algebraic/combinatorial structure underlying in various areas in mathematics.

Let us briefly summarize the main result of IN14 highlighting some key words. We study the WKB solutions of the Schrödinger equation on a compact Riemann surface. The principal term of the potential of the Schrödinger equation determines a quadratic differential on the surface, and the latter further determines a graph on the surface called the Stokes graph. The Stokes graph plays a central role to study the local and global analytic properties of the WKB solutions, and the Voros symbols describe the monodoromy data of the WKB solutions. Under a continuous deformation of the potential, the Stokes graph may change its topology. We call this phenomenon the mutation of Stokes graphs. Meanwhile, the Stokes graph induces a triangulation of a bordered surface with marked points in the sense of FST08. Furthermore, the mutation of Stokes graphs corresponds to the mutation of triangulations called signed flips and signed pops. These facts provide a natural bridge between exact WKB analysis and cluster algebra theory with the help of the surface realization of cluster algebras developed by GSV05, FG06, FST08, FT12. Along the mutation of Stokes graphs, the Voros symbols also mutate (or jump) due to the Stokes phenomenon. The main result of [IN14 is that the Voros symbols

2010 Mathematics Subject Classification. 13F60, 34M60. 
mutate as the variables of the corresponding cluster algebra. To be more precise, they mutate by signed mutations and signed pops of the extended seeds, which are certain extensions of the ordinary mutation of cluster algebras.

In IN14 it was technically essential to assume that the Schrödinger equation has no turning points of simple-pole type (simple-pole for short). In this paper we lift this restriction and treat the case where there are simple poles. The connection formula of the WKB solutions around a simple pole was obtained by Koike Koi00. This is the counterpart of the celebrated Voros' connection formula of the WKB solutions around a usual turning point. Based on Koike's connection formula, we obtain the following results in this paper.

(a). Firstly, we obtain the Delabaere-Dillinger-Pham (DDP) type jump formula for the Voros symbols at the reduction of a Stokes segment involving a simple pole (Theorem 2.17). Our formula is closely related to the result of Kamimoto et al. [KKKT10] (see Remark 2.18).

(b). Secondly, we reformulate the above jump formula in view of cluster algebra theory, where we have two novel features in contrast to the previous case in [IN14.

(1). Instead of using the surface realization of cluster algebras, we need to use the orbifold realization of cluster algebras by Felikson, Shapiro, and Tumarkin [FST11]. The simple poles correspond to the orbifold points therein.

(2). The Voros symbols mutate as the variables of generalized cluster algebras introduced by Chekhov and Shapiro CS14 instead of ordinary cluster algebras (Theorem 4.6). To be more precise, they mutate by signed mutations, which are certain extensions of the ordinary mutation of generalized cluster algebras.

The organization of the paper is as follows. In Section 2 we consider the Schrödinger equation on a compact Riemann surface especially having simple poles. Then, we obtain the DDP type jump formula for the Voros symbols at the reduction of a Stokes segment involving a simple pole. In Section 3 we show how the above reduction is related to the signed flips of labeled Stokes triangulations of an orbifold. In Section 4 we reformulate our jump formula in view of the above correspondence to Stokes triangulations, and show that the Voros symbols mutate as the variables of generalized cluster algebras at the mutation of Stokes graphs involving simple poles.

Acknowledgements. We greatly thank Dylan Thurston, who suggested us the link between simple poles and orbifold points at the workshop "Cluster algebras and related topics" held at Mathematisches Forschungsinstitut Oberwolfach in December, 2013. We also thank Anna Felikson, Anne-Sophie Gleitz, Takahiro Kawai, Tatsuya Koike, Michael Shapiro and Yoshitsugu Takei for useful communications and discussions. K.I is supported by Research Fellowships of JSPS KAKENHI Grant Number 13J02831.

\section{Exact WKB analysis of SchröDinger EQuation With Simple POlES}

2.1. Schrödinger equation and assumptions. Let us consider a Schrödinger equation (i.e., a second order linear differential equation containing a large parameter $\eta$ which corresponds to the inverse of the Planck constant $\hbar$ ) for a complex 
function $((-1 / 2)$-form, to be precise) $\psi(z, \eta)$ defined on a compact connected Riemann surface $\Sigma$ which also depends on $\eta$ :

$$
\begin{gathered}
\left(\frac{d^{2}}{d z^{2}}-\eta^{2} Q(z, \eta)\right) \psi(z, \eta)=0, \\
Q(z, \eta)=Q_{0}(z)+\eta^{-2} Q_{2}(z) .
\end{gathered}
$$

Here (2.1) is a local expression of our equation in a local coordinate $z$ of $\Sigma$. The coefficients $Q_{0}(z), Q_{2}(z)$ are meromorphic functions. If we take a coordinate transformation $z=z(\tilde{z})$, we obtain an equation of the same form as (2.1) for the coordinate $\tilde{z}$ as follows:

$$
\begin{gathered}
\left(\frac{d^{2}}{d \tilde{z}^{2}}-\eta^{2} \tilde{Q}(\tilde{z}, \eta)\right) \tilde{\psi}=0, \quad \tilde{\psi}(\tilde{z}, \eta)=\psi(z(\tilde{z}), \eta)\left(\frac{d z(\tilde{z})}{d \tilde{z}}\right)^{-1 / 2}, \\
\tilde{Q}(\tilde{z}, \eta)=Q(z(\tilde{z}), \eta)\left(\frac{d z(\tilde{z})}{d \tilde{z}}\right)^{2}-\frac{1}{2} \eta^{-2}\{z(\tilde{z}) ; \tilde{z}\}
\end{gathered}
$$

Here $\{z(\tilde{z}) ; \tilde{z}\}$ is the Schwarzian derivative:

$$
\{z(\tilde{z}) ; \tilde{z}\}=\left(\frac{d^{3} z(\tilde{z})}{d \tilde{z}^{3}} / \frac{d z(\tilde{z})}{d \tilde{z}}\right)-\frac{3}{2}\left(\frac{d^{2} z(\tilde{z})}{d \tilde{z}^{2}} / \frac{d z(\tilde{z})}{d \tilde{z}}\right)^{2} .
$$

In particular, the transformation law

$$
\tilde{Q}_{0}(\tilde{z})=Q_{0}(z(\tilde{z}))\left(\frac{d z}{d \tilde{z}}\right)^{2}
$$

of the principal terms of the potential of the Schrödinger equations coincides with that of a meromorphic quadratic differential, that is, a meromorphic section of the line bundle $\omega_{\Sigma}^{\otimes 2}$, where $\omega_{\Sigma}$ is the holomorphic cotangent bundle on $\Sigma$.

Definition 2.1. The quadratic differential associated with the Schrödinger equation (2.1) is the meromorphic quadratic differential on $\Sigma$ which is locally given by

$$
\phi=Q_{0}(z) d z^{\otimes 2} .
$$

Here $Q_{0}(z)$ is the principal term of the potential $Q(z, \eta)$ of the Schrödinger equation in a local coordinate $z$.

The zeros and simple poles of $\phi$ play important roles in exact WKB analysis.

Definition 2.2 ([KT05, Definition 2.6], Koi00). Let $\phi$ be the quadratic differential defined by (2.5).

- Turning points of (2.1) are zeros of $\phi$.

- Turning points of simple-pole type of (2.1) are simple poles of $\phi$. We call these points simple poles of (2.1) to distinguish them from the usual turning points defined above.

The set of zeros, simple poles and poles of order $\geq 2$ of $\phi$ are denoted by $P_{0}, P_{\mathrm{s}}$ and $P_{\infty}$, respectively. We also set $P=P_{0} \cup P_{\mathrm{s}} \cup P_{\infty}$.

In our previous paper [IN14] which disclosed a relationship between exact WKB analysis and cluster algebras, we assumed that $\phi$ does not have any simple poles. In this paper we will extend the results of IN14 to the case that $\phi$ may have simple poles.

We impose a similar assumption as [N14] for the potential $Q(z, \eta)$ in (2.1). 
Assumption 2.3. Let $\phi$ be the quadratic differential associated with (2.1).

- $\phi$ has at least one zero, and at least one pole.

- All zeros of $\phi$ are simple.

- Suppose that a point $p \in \Sigma$ is a pole of $Q_{2}(z)$. Then, $p$ is a pole of $\phi$.

- For any simple pole $s, Q_{2}(z)$ has a pole of order at most 2 at $s$.

- Suppose that a point $p \in P_{\infty}$ is a pole of $\phi$ of order 2 , and $z$ be a local coordinate around $p$ satisfying $z(p)=0$. Then, $Q_{2}(z)$ has a pole of order 2 at $z=0$ and satisfies

$$
Q_{2}(z)=-\frac{1}{4 z^{2}}(1+O(z)) \text { as } z \rightarrow 0
$$

- Suppose that a point $p \in P_{\infty}$ is a pole of $\phi$ of order $m \geq 3$. Then, $Q_{2}(z)$ may have pole at $p$ and

$$
\text { (pole order of } \left.Q_{2}(z) \text { at } p\right)<1+\frac{m}{2} \text {. }
$$

2.2. WKB solutions. For the equation (2.1), we can construct a pair of formal solutions, called the WKB solutions, in the following form (see [KT05, Section 2]):

$$
\psi_{ \pm}(z, \eta)=\frac{1}{\sqrt{S_{\text {odd }}(z, \eta)}} \exp \left( \pm \int^{z} S_{\text {odd }}(z, \eta) d z\right)
$$

where $S_{\text {odd }}(z, \eta)$ is a formal (Laurent) series in $\eta^{-1}$ defined as the odd part of the formal solution $S(z, \eta)=\eta S_{-1}(z)+S_{0}(z)+\eta^{-1} S_{1}(z)+\cdots$ of the Riccati equation

$$
\frac{d S}{d z}+S^{2}=\eta^{2} Q(z, \eta)
$$

associated with (2.1). Namely, if we denote by $S^{( \pm)}(z, \eta)= \pm \eta \sqrt{Q_{0}(z)}+\cdots$ the two formal solutions of (2.9), then their odd part and even part are defined by

$S_{\text {odd }}(z, \eta)=\frac{1}{2}\left(S^{(+)}(z, \eta)-S^{(-)}(z, \eta)\right), \quad S_{\text {even }}(z, \eta)=\frac{1}{2}\left(S^{(+)}(z, \eta)+S^{(-)}(z, \eta)\right)$.

It is known that the (formal series valued) 1-form $S_{\text {odd }}(z, \eta) d z$ transforms as

$$
\tilde{S}_{\text {odd }}(\tilde{z}, \eta)=S_{\text {odd }}(z(\tilde{z}), \eta) \frac{d z(\tilde{z})}{d \tilde{z}}
$$

under the coordinate transformation $z=z(\tilde{z})$. (See [KT05, Corollary 2.17], IN14, Proposition $2.7(\mathrm{~b})]$.) Here $\tilde{S}_{\text {odd }}(\tilde{z}, \eta)$ is the odd part of the formal solution of the Riccati equation associated with (2.2). Therefore, the 1 -form $S_{\text {odd }}(z, \eta) d z$ is globally defined (but multi-valued) on $\Sigma \backslash P$, and its integrals are independent of the choice of the local coordinate.

Each coefficient of $S_{\text {odd }}(z, \eta)$ may have singularities at points in $P$. However, for any $a \in P_{0}$ (resp., for any $s \in P_{\mathrm{s}}$ ), we can define the integral

$$
\int_{a}^{z} S_{\text {odd }}(z, \eta) d z \quad\left(\text { resp., } \int_{s}^{z} S_{\text {odd }}(z, \eta) d z\right)
$$

in the sense of contour integral (see [KT05, Section 2]). On the other hand, we cannot define an integral of $S_{\text {odd }}(z, \eta) d z$ from any point $p \in P_{\infty}$ because the principal term $\eta \sqrt{Q_{0}(z)} d z$ is singular at $p$. However, subtracting the principal term, we can define an integral from $p$ due to Assumption 2.3 
Proposition 2.4 ([IN14, Proposition 2.8]). Under Assumption [2.3, for any point $p \in P_{\infty}$ and any local coordinate $z$ of $\Sigma$ around $p$ such that $z=0$ at $p$, the formal power series valued 1-form

$$
S_{\text {odd }}^{\mathrm{reg}}(z, \eta) d z:=\left(S_{\text {odd }}(z, \eta)-\eta \sqrt{Q_{0}(z)}\right) d z
$$

is integrable at $z=0$. Namely, for any $n \geq 0$, there exists a real number $\ell>-1$ such that

$$
S_{\text {odd }, n}(z)=O\left(z^{\ell}\right) \quad \text { as } z \rightarrow 0,
$$

where $S_{\text {odd }, n}(z)$ is the coefficient of $\eta^{-n}$ in $S_{\text {odd }}^{\text {reg }}(z, \eta)$. Moreover, all coefficients of $S_{\text {odd }}^{\text {reg }}(z, \eta)$ are holomorphic at $p$ if it is an even order pole of $\phi$.

We call $S_{\text {odd }}^{\mathrm{reg}}(z, \eta)$ the regular part of $S_{\text {odd }}(z, \eta)$. Proposition 2.4 implies that the integral

$$
\int_{p}^{z} S_{\text {odd }}^{\mathrm{reg}}(z, \eta) d z=\int_{p}^{z}\left(S_{\text {odd }}(z, \eta)-\eta \sqrt{Q_{0}(z)}\right) d z
$$

from any point $p \in P_{\infty}$ is well-defined.

2.3. Borel resummation method. By the formal series expansion, we have

$$
\psi_{ \pm}(z, \eta)=\exp \left( \pm \eta \int^{z} \sqrt{Q_{0}(z)} d z\right) \eta^{-1 / 2} \sum_{k=0}^{\infty} \eta^{-k} \psi_{ \pm, k}(z)
$$

The expansion (2.14) is a divergent series of $\eta^{-1}$ in general. To give (2.14) an analytic interpretation, we employ the Borel resummation method (for a formal series of $\eta$ ). For the convenience of the readers, we give a rough definition of Borel resummation method. (See Cos09] for details.)

Definition 2.5 (e.g., [KT05, §1]). If the Laplace integral

$$
\mathcal{S}\left[\psi_{ \pm}\right](z, \eta)=\int_{\mp \mathfrak{s}(z)}^{\infty} e^{-\eta y} \psi_{ \pm, B}(z, y) d y
$$

is well-defined and becomes an analytic function of sufficiently large $\eta>0$, and also becomes a holomorphic function of $z$ in a neighborhood of a point $z_{0} \in \Sigma \backslash P$, we call (2.15) the Borel sum of the WKB solution $\psi_{ \pm}(z, \eta)$. Here $\mathfrak{s}(z)=\int{ }^{z} \sqrt{Q_{0}(z)} d z$ and

$$
\psi_{ \pm, B}(z, y)=\sum_{k=1}^{\infty} \frac{\psi_{ \pm, k}(z)}{\Gamma(k+1 / 2)}(y \pm \mathfrak{s}(z))^{k-1 / 2}
$$

is the Borel transform of $\psi_{ \pm}(z, \eta)$. The path of the integral (2.15) is taken along a straight line parallel to the positive real axis.

We also use a simplified notation $\Psi_{ \pm}=\mathcal{S}\left[\psi_{ \pm}\right]$for the Borel sums of the WKB solutions. The convergence of the Borel transform $\psi_{ \pm, B}(z, y)$ near $y=\mp \mathfrak{s}(z)$ can be shown easily ([KT05, Lemma 2.5]). The Borel summability of $\psi_{ \pm}(z, \eta)$ demands that $\psi_{ \pm, B}(z, y)$ can be extended to an analytic function of $y$ on a domain containing $\{y \in \mathbb{C} \mid \operatorname{Re}(y) \geq 0\}$, and grows at most exponentially when $|y| \rightarrow+\infty$ on the domain. If the Borel sum is well-defined, it is asymptotically expanded to the original WKB solution as $\eta \rightarrow+\infty$. 
2.4. Stokes graphs, Stokes segments and Borel summability. Many properties of the WKB solutions, such as the Borel summability (i.e., well-definedness of the Borel sum (2.15)), can be read off from the geometry of Stokes graph, which is a graph described by trajectories of the quadratic differential $\phi$ defined in (2.5). Here a trajectory of a quadratic differential $f(z) d z^{\otimes 2}$ is a leaf of the foliation on $\Sigma$ defined by the equation

$$
\operatorname{Im} \int^{z} \sqrt{f(z)} d z=\text { constant. }
$$

See [Str84] and [BS13] for properties of trajectories of quadratic differentials.

Definition 2.6 (KT05, Definition 2.6], [Koi00]). Let $\phi$ be the quadratic differential associated with (2.1). A Stokes curve of (2.1) is a trajectory of $\phi$ one of whose endpoints is a turning point or a simple pole of (2.1). Namely, in a local coordinate $z$ on $\Sigma$, Stokes curves emanating from $a \in P_{0} \cup P_{\mathrm{s}}$ are defined as

$$
\operatorname{Im} \int_{a}^{z} \sqrt{Q_{0}(z)} d z=0 .
$$

In the whole of the article, we assume the following:

Assumption 2.7. The quadratic differential $\phi$ associated with (2.1) has no recurrent trajectory. Here a recurrent trajectory is a trajectory which is not a closed trajectory and has the limit set consisting of more than one point in at least one direction (see [BS13, Section 3.4]).

Under Assumption 2.7, any Stokes curve emanates from a turning point or a simple pole flows into a pole of $\phi$ or a turning point (see [BS13, Section 3.4]). In other words, turning points, poles of $\phi$ and Stokes curves define a graph on the Riemann surface $\Sigma$.

Definition 2.8. $\quad$ - The Stokes graph of (2.1) is a graph on $\Sigma$ whose vertices consist of zeros and poles of $\phi$, and whose edges are Stokes curves.

- Faces of the Stokes graph are called Stokes regions.

The Stokes graph is defined only from the principal term $Q_{0}(z)$ in the potential (or from the quadratic differential $\phi$ ). We sometimes write $G=G(\phi)$ for the Stokes graph when we emphasize the dependence on $\phi$.

Figure 1depicts examples of Stokes graphs on $\mathbb{P}^{1}$. From each turning point (resp., simple pole) three Stokes curves (resp., one Stokes curve) emanate. Trajectories behave as in Figure 2 near a turning point and a simple pole. In what follows, we use the symbols $\times$ and $\otimes$ for a turning point and a simple pole, respectively. Also, the symbol $\bullet$ is used to represent a point in $P_{\infty}$.

Definition 2.9. - A Stokes curve connecting points $a$ and $b\left(a, b \in P_{0} \cup P_{\mathrm{s}}\right)$ is called a Stokes segment.

- If the Stokes graph does not contain any Stokes segment, the Stokes graph (or the quadratic differential $\phi$ ) is said to be saddle-free.

A Stokes segment is nothing but a saddle trajectory of $\phi$ (see [Str84]). Typical examples of Stokes segments are shown in Figure 3 .

- A Stokes segment of type $I$ connects two distinct turning points $a_{1}$ and $a_{2}$. Figure 1 (e) depicts an example of a type I Stokes segment. 


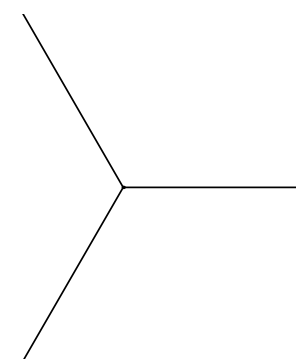

(a) : z.

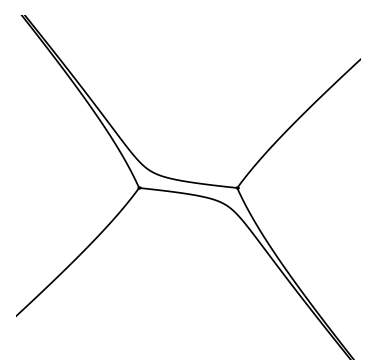

(d) $: e^{+i \pi / 10}\left(1-z^{2}\right)$.

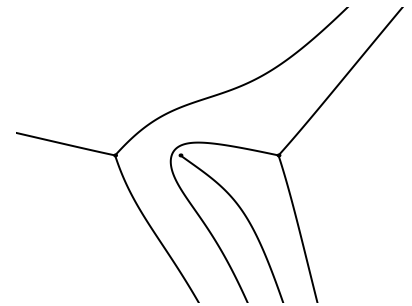

(g) : $-\frac{e^{+\pi i / 5}(z-2)(z+3)}{z+1}$

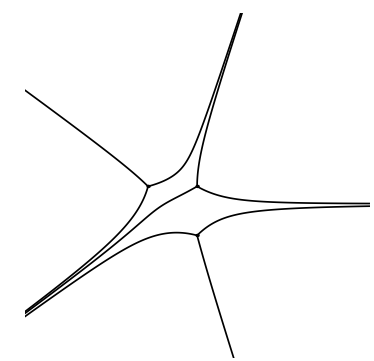

(b) : $z(z+1)(z+i)$.

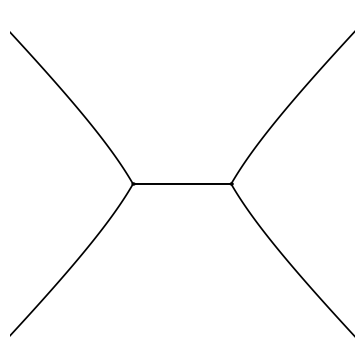

(e) : $1-z^{2}$.

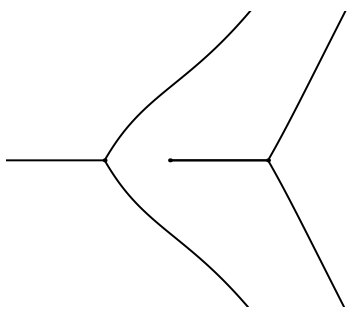

(h) : $-\frac{(z-2)(z+3)}{z+1}$

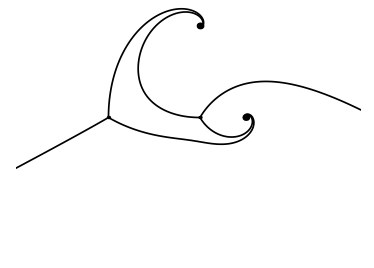

(c) : $\frac{z(z+2)}{(z-1)^{2}(z-2 i)^{2}}$.

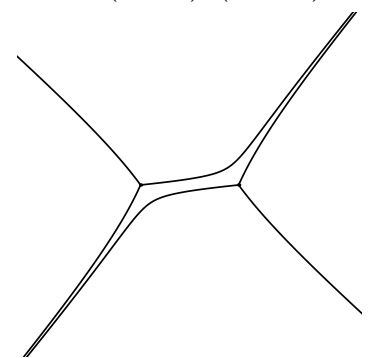

(f) $: e^{-i \pi / 10}\left(1-z^{2}\right)$.

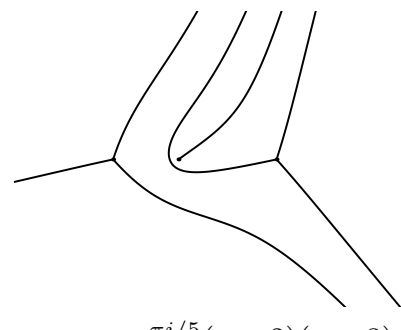

(i) : $-\frac{e^{-\pi i / 5}(z-2)(z+3)}{z+1}$

Figure 1. Examples of Stokes graphs. The rational functions represent $Q_{0}(z)$.

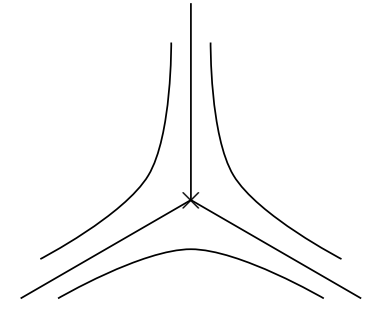

(a) around a turning point

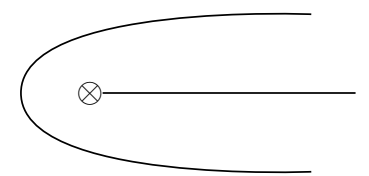

(b) around a simple pole

FIgURE 2. Foliations around a turning point and a simple pole. 


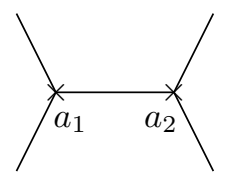

type I

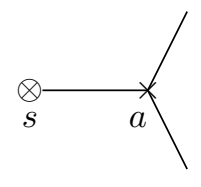

type II

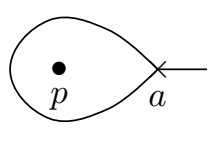

type III

Figure 3. Examples of Stokes segments. Each figure designates a part of the Stokes graph.

- A Stokes segment of type II connects a simple pole $s$ and a turning point a. Figure 1 (h) depicts an example of a type II Stokes segment.

- A Stokes segment of type III forms a closed loop whose end point is a turning point $a$. It appears around a double pole $p$ of $\phi$.

Theorem 2.10 ([KS $)$. Suppose that the potential $Q(z, \eta)$ of (2.1) satisfies Assumptions 2.3 and 2.7. If a path $\beta$ on $\Sigma \backslash\left(P_{0} \cup P_{\mathrm{s}}\right)$ never intersects with any Stokes segment in the Stokes graph, then the formal power series $\int_{\beta} S_{\mathrm{odd}}^{\mathrm{reg}}(z, \eta) d z$ is Borel summable. Moreover, if the Stokes graph is saddle-free, then the WKB solutions are Borel summable on each Stokes region. The Borel sum of the WKB solutions give analytic solutions of the Schrödinger equation (2.1), and they are asymptotically expanded to the original WKB solutions when $\eta \rightarrow+\infty$.

Remark 2.11. Theorem 2.10 was proved in $[\mathrm{KS}]$ when the underlying Riemann surface $\Sigma$ is the Riemann sphere $\mathbb{P}^{1}$. Since the proof by [KS] only use local properties in a Stokes region, the statement can be generalized to the case where $\Sigma$ is a general compact Riemann surface.

As is inferred from Theorem 2.10, the existence of a Stokes segment may break the Borel summability of the WKB solutions. Furthermore, a Stokes segment causes a "jump" for the WKB solutions etc. (See [Vor83, Section 7]; see also Section 2.7 below.)

2.5. Connection formulas on Stokes curves. In this subsection we assume that the Stokes graph $G$ is saddle-free. Then, by Theorem 2.10, we have the Borel sums of the WKB solutions on each Stokes region. Here we discuss connection formulas describing the relations between the Borel sums given in adjacent Stokes regions. The connection formulas will be used in the proof of Theorem 2.17 below.

First, we recall Voros' connection formula on a Stokes curve emanating from a turning point. Let $a \in P_{0}$ be a turning point, and $C$ be a Stokes curve emanating from $a$. Take any two points $z_{1}$ and $z_{2}$ near $C$ satisfying the following conditions (see Figure $4(\mathrm{a})$ ):

- $z_{1}$ and $z_{2}$ are contained in some Stokes regions $D_{1}$ and $D_{2}$, respectively. The Stokes curve $C$ is a common boundary of $D_{1}$ and $D_{2}$.

- $D_{2}$ comes next to $D_{1}$ in the anticlockwise direction with the reference point $a$.

- The line segment connecting $z_{1}$ and $z_{2}$ intersects with the Stokes graph at exactly one point $z_{0}$ which lies on $C$. 


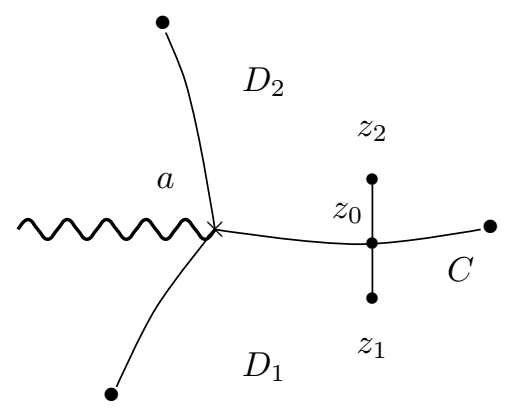

(a) : $C$ emanates from $a \in P_{0}$.

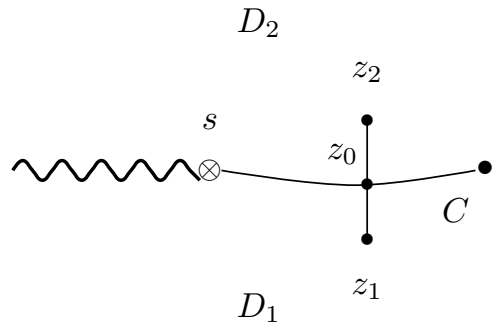

(b) : $C$ emanates from $s \in P_{\mathrm{s}}$.

Figure 4. Connection problems on Stokes curves. The wiggly lines designate branch cuts to define the branch of $\sqrt{Q_{0}(z)}$.

Take the WKB solutions normalized at the turning point a (see [KT05, Section 2]):

$$
\psi_{ \pm, a}(z, \eta)=\frac{1}{\sqrt{S_{\text {odd }}(z, \eta)}} \exp \left( \pm \int_{a}^{z} S_{\text {odd }}(z, \eta) d z\right) .
$$

Here we assume that the integral in (2.18) is defined for $z$ which lies in a small neighborhood of $z_{1}$ or $z_{2}$, and the path from $a$ to $z$ is given by a composition of the following two paths; one is the path from $a$ to $z_{0}$ along $C$, and the other one is the straight path from $z_{0}$ to $z$. In order to fix the normalization (2.18) completely, we fix a branch of the square root $\sqrt{Q_{0}(z)}$ on $C$ after taking a branch cut as indicated Figure 4 (a). For $j=1,2$, let $\Psi_{ \pm, a}^{D_{j}}$ be the Borel sum of $\psi_{ \pm, a}$ defined in a neighborhood of $z_{j}$ included in the Stokes region $D_{j}$. We also denote by the same symbol $\Psi_{ \pm, a}^{D_{j}}$ its analytic continuation to the whole $D_{j}$. Under the situation, we have the following connection formula.

Theorem 2.12 ([Vor83, Section 6], [AKT91, Section 2]). The analytic continuations of $\Psi_{ \pm, a}^{D_{1}}$ to $D_{2}$ across the Stokes curve $C$ satisfy one of the following equations:

$$
(i):\left\{\begin{array}{l}
\Psi_{+, a}^{D_{1}}=\Psi_{+, a}^{D_{2}}+i \Psi_{-, a}^{D_{2}} \\
\Psi_{-, a}^{D_{1}}=\Psi_{-, a}^{D_{2}} .
\end{array} \quad(i i):\left\{\begin{array}{l}
\Psi_{+, a}^{D_{1}}=\Psi_{+, a}^{D_{2}} \\
\Psi_{-, a}^{D_{1}}=\Psi_{-, a}^{D_{2}}+i \Psi_{+, a}^{D_{2}} .
\end{array}\right.\right.
$$

Here the case (i) occurs when $\operatorname{Re}\left(\int_{a}^{z} \sqrt{Q_{0}(z)} d z\right)>0$ on $C$, while the case (ii) occurs when $\operatorname{Re}\left(\int_{a}^{z} \sqrt{Q_{0}(z)} d z\right)<0$ on $C$.

A similar connection formula on a Stokes curve emanating from a simple pole is discovered by Koike. Suppose that we have the same situation as above after replacing "a turning point $a$ " to "a simple pole $s$ " as indicated in Figure 4 (b). Take the WKB solutions normalized at the simple pole $s$ (지에, Section 2])

$$
\psi_{ \pm, s}(z, \eta)=\frac{1}{\sqrt{S_{\text {odd }}(z, \eta)}} \exp \left( \pm \int_{s}^{z} S_{\text {odd }}(z, \eta) d z\right),
$$


and define their Borel sums $\Psi_{ \pm, s}^{D_{j}}$ defined on $D_{j}(j=1,2)$, by the same manner as above. Then, we have the following connection formula.

Theorem 2.13 ( Koi00, Theorem 2.1]). The analytic continuations of $\Psi_{ \pm, a}^{D_{1}}$ to $D_{2}$ across the Stokes curve $C$ satisfy one of the following equations:
$(i):\left\{\begin{array}{l}\Psi_{+, s}^{D_{1}}=\Psi_{+, s}^{D_{2}}+i\left(t+t^{-1}\right) \Psi_{-, s}^{D_{1}} \\ \Psi_{-, s}^{D_{1}}=\Psi_{-, s}^{D_{2}} .\end{array}\right.$
$(i i):\left\{\begin{array}{l}\Psi_{+, s}^{D_{1}}=\Psi_{+, s}^{D_{2}} \\ \Psi_{-, s}^{D_{1}}=\Psi_{-, s}^{D_{2}}+i\left(t+t^{-1}\right) \Psi_{+, s}^{D_{2}} .\end{array}\right.$

Here

$$
t=t(s)=\exp (\pi i \sqrt{1+4 b(s)}), \quad b(s)=\lim _{z \rightarrow s}\left((z-s)^{2} Q_{2}(z)\right),
$$

and the case (i) occurs when $\operatorname{Re}\left(\int_{s}^{z} \sqrt{Q_{0}(z)} d z\right)>0$ on $C$, while the case (ii) occurs when $\operatorname{Re}\left(\int_{s}^{z} \sqrt{Q_{0}(z)} d z\right)<0$ on $C$.

Remark 2.14. Theorems 2.12 and 2.13 are proved by Vor83, AKT91 and Koi00, respectively, in the case that $\Sigma=\mathbb{P}^{1}$ and the potential is a rational function. The proofs of [AKT91] and Koi00] are based on a formal local transformation to a certain canonical equation near a turning point and a simple pole. (We also need the Borel summability of the formal transformation series, which follows from the Borel summability of the WKB solutions as is shown in KK11.) Since the transformations are constructed locally, the same discussion of [AKT91] and [Koi00] is applicable to case that $\Sigma$ is a general compact Riemann surface. Therefore, together with Theorem 2.10. Theorems 2.12 and 2.13 are valid when $\Sigma$ is a general compact Riemann surface.

The connection formulas in Theorems 2.12 and 2.13 are quite effective for the study of global properties of solutions of the Schrödinger equation. See KT05, Section 3] for computation of the monodromy of a Fuchsian Schrödinger equation via the above connection formulas.

2.6. Voros symbols. Here we introduce an important notion, called Voros symbols. Voros symbols are formal series defined as the integral of $S_{\text {odd }}(z, \eta) d z$ along a path or a cycle in the Riemann surface $\hat{\Sigma}$ of $\sqrt{\phi}$. The Riemann surface $\hat{\Sigma}$ is a double cover of $\Sigma$ branching at odd order zeros and odd order poles of $\phi$.

Denote by $\hat{P}_{0}, \hat{P}_{\mathrm{S}}$ and $\hat{P}_{\infty}$ the lift of $P_{0}, P_{\mathrm{S}}$ and $P_{\infty}$ on $\hat{\Sigma}$, respectively. We also set $\hat{P}=\hat{P}_{0} \cup \hat{P}_{\mathrm{s}} \cup \hat{P}_{\infty}$ and

$$
\begin{aligned}
H_{1}\left(\hat{\Sigma} \backslash\left(\hat{P}_{0} \cup \hat{P}_{\mathrm{s}}\right), \hat{P}_{\infty}\right) & :=H_{1}\left(\hat{\Sigma} \backslash\left(\hat{P}_{0} \cup \hat{P}_{\mathrm{s}}\right), \hat{P}_{\infty} ; \mathbb{Z}\right), \\
H_{1}(\hat{\Sigma} \backslash \hat{P}) & :=H_{1}(\hat{\Sigma} \backslash \hat{P} ; \mathbb{Z}) .
\end{aligned}
$$

Here the one in (2.23) is a relative homology group. We call an element $\beta \in$ $H_{1}\left(\hat{\Sigma} \backslash\left(\hat{P}_{0} \cup \hat{P}_{\mathrm{s}}\right), \hat{P}_{\infty}\right)$ and $\gamma \in H_{1}(\hat{\Sigma} \backslash \hat{P})$ a path and a cycle, respectively, to distinguish them. There is a natural inclusion $H_{1}(\hat{\Sigma} \backslash \hat{P}) \hookrightarrow H_{1}\left(\hat{\Sigma} \backslash\left(\hat{P}_{0} \cup \hat{P}_{\mathrm{s}}\right), \hat{P}_{\infty}\right)$.

Definition 2.15 (DDP93, Section 2.1]). - Let $\beta \in H_{1}\left(\hat{\Sigma} \backslash\left(\hat{P}_{0} \cup \hat{P}_{\mathrm{s}}\right), \hat{P}_{\infty}\right)$ be a path. The formal power series

$$
W_{\beta}(\eta)=\int_{\beta} S_{\text {odd }}^{\mathrm{reg}}(z, \eta) d z
$$


is called the Voros coefficient for the path $\beta$. (Note that the integral of $S_{\text {odd }}^{\text {reg }}(z, \eta) d z$ along a path $\beta$ is well-defined by Proposition 2.4.) The formal series $e^{W_{\beta}(\eta)}$ is called the Voros symbol for the path $\beta$.

- Let $\gamma \in H_{1}(\hat{\Sigma} \backslash \hat{P})$ be a cycle. The formal series

$$
V_{\gamma}(\eta)=\oint_{\gamma} S_{\text {odd }}(z, \eta) d z
$$

is called the Voros coefficient for the cycle $\gamma$. The formal series $e^{V_{\gamma}(\eta)}$ is called the Voros symbol for the cycle $\gamma$.

Theorem 2.10 gives a criterion for the Borel summability of the Voros symbols.

Proposition 2.16 ([KS], see also [IN14, Corollary 2.21]). If a path $\beta \in H_{1}(\hat{\Sigma} \backslash$ $\left.\left(\hat{P}_{0} \cup \hat{P}_{\mathrm{s}}\right), \hat{P}_{\infty}\right)$ (resp., a cycle $\left.\gamma \in H_{1}(\hat{\Sigma} \backslash \hat{P})\right)$ never intersects with a Stokes segment in the Stokes graph $G$, then the Voros symbol for the path $\beta$ (resp., for the cycle $\gamma$ ) is Borel summable. In particular, if the Stokes graph $G$ is saddle-free, all Voros symbols are Borel summable.

The Borel sums of Voros symbols naturally appear in the expression of global connection formula for the WKB solutions ([KT05, Section 3]; see also Section 2.8 below).

2.7. Mutation of Stokes graphs and jump formula for Voros symbols. The saddle-free condition is essential in the above results. Now we discuss effects of Stokes segments. Let us consider the situation that the Stokes graph $G(\phi)$ has a unique Stokes segment $\ell_{0}$. We restrict our discussion to the case that $\ell_{0}$ is of type II in Figure 3. (The Stokes segment of type I (resp., type III) has already been analyzed by [DDP93, AKT09] (resp., by [AIT, IN14]).)

For the purpose, following [IN14, Section 3.6], let us consider the $S^{1}$-family of potentials of the Schrödinger equation defined by

$$
Q^{(\theta)}(z, \eta)=e^{2 i \theta} Q\left(z, e^{i \theta} \eta\right) \quad(\theta \in \mathbb{R}) .
$$

Here $Q(z, \eta)=Q^{(0)}(z, \eta)$ is the original potential of (2.1). Denote by

$$
G_{\theta}=G\left(e^{2 i \theta} \phi\right)
$$

the Stokes graph of the Schrödinger equation with the potential (2.27). Here we assume that the potential (2.27) satisfies Assumption 2.7 for any $\theta$.

The $S^{1}$-family (2.27) gives a reduction of the Stokes segment in the following sense. Note that, if a Stokes segment appears in the original Stokes graph $G_{0}=$ $G(\phi)$ and it connects two points $a, b \in P_{0} \cup P_{\mathrm{s}}$, then the equality

$$
\int_{a}^{b} \sqrt{Q_{0}(z)} d z \in \mathbb{R}_{\neq 0}
$$

holds by the definition (2.17) of a Stokes curve. Hence, for any sufficiently small $\delta>0$, the Stokes segment $\ell_{0}$ is reduced (to two Stokes curves) in the Stokes graphs $G_{ \pm \delta}=G\left(e^{ \pm 2 i \delta} \phi\right)$, and they become saddle-free (c.f., [BS13, Section 5]). Moreover, the topology of $G_{+\delta}$ and that of $G_{-\delta}$ are different as in Figure 5. We call such a discontinuous change of the topology of Stokes graphs (caused by a reduction of a Stokes segment) the mutation of Stokes graphs. This is a key phenomenon that relates exact WKB analysis to cluster algebras. 

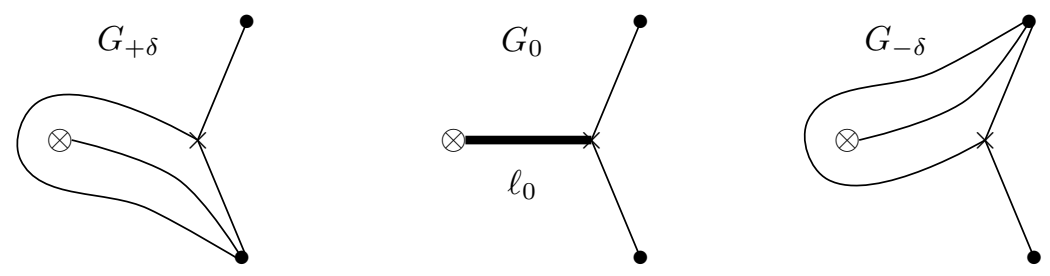

Figure 5. Reduction of a Stokes segment of type II and the mutation of Stokes graphs. The thick line designates the Stokes segment.

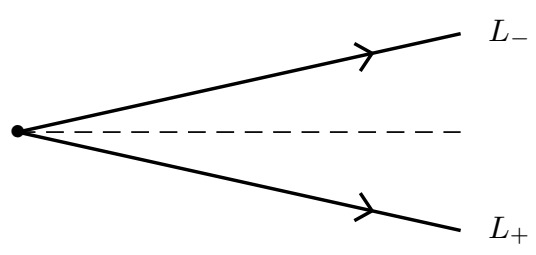

Figure 6 . Rotated paths of Laplace integral for $\mathcal{S}_{ \pm}[\bullet]$ on $y$-plane.

The dashed line represents the half line with no angle.

Denote by $e^{W_{\beta}^{(\theta)}}=e^{W_{\beta}^{(\theta)}(\eta)}$ and $e^{V_{\gamma}^{(\theta)}}=e^{V_{\gamma}^{(\theta)}(\eta)}$ the Voros symbols for the Schrödinger equation with the potential (2.27). As is shown in [IN14, Lemma 3.8], we have

$$
e^{W_{\beta}^{(\theta)}(\eta)}=e^{W_{\beta}\left(e^{i \theta} \eta\right)}, \quad e^{V_{\gamma}^{(\theta)}(\eta)}=e^{V_{\gamma}\left(e^{i \theta} \eta\right)},
$$

where $e^{W_{\beta}(\eta)}$ and $e^{V_{\gamma}(\eta)}$ are the Voros symbols of the original equation (2.1). (Note that the Riemann surfaces $\hat{\Sigma}^{(\theta)}$ of $e^{i \theta} \sqrt{\phi}$ are common for all $\theta$. Hence we can identify the paths and cycles on $\hat{\Sigma}^{(\theta)}$ for different $\theta$ naturally.) Since the Stokes graphs $G_{ \pm \delta}$ are saddle-free, Proposition 2.16 implies that $e^{W_{\beta}^{( \pm \delta)}}$ and $e^{V_{\gamma}^{( \pm \delta)}}$ are Borel summable for any sufficiently small $\delta>0$. As is shown in [IN14, Section 3.6], the limits $\delta \rightarrow+0$ of these Borel sums exist, and they are given by

$$
\lim _{\delta \rightarrow+0} \mathcal{S}\left[e^{W_{\beta}^{( \pm \delta)}}\right](\eta)=\mathcal{S}_{ \pm}\left[e^{W_{\beta}}\right](\eta), \quad \lim _{\delta \rightarrow+0} \mathcal{S}\left[e^{V_{\gamma}^{( \pm \delta)}}\right](\eta)=\mathcal{S}_{ \pm}\left[e^{V_{\gamma}}\right](\eta) .
$$

Here $\mathcal{S}_{ \pm}[\bullet]$ means the Borel sum in the direction $\pm \varepsilon$ for a sufficiently small $\varepsilon>0$; it is defined as a Laplace integral of the same form as (2.15) whose integration path is taken along a half line $L_{ \pm}$, which has a small angle $\mp \varepsilon$ as indicated in Figure 6. The Borel sums $\mathcal{S}_{ \pm}\left[e^{W_{\beta}}\right]$ and $\mathcal{S}_{ \pm}\left[e^{V_{\gamma}}\right]$ give analytic functions of $\eta$ defined on $\{\eta \in \mathbb{R} \mid \eta \gg 1\}$.

What is important here is that, the mutation of Stokes graphs yields a discrepancy (or jump) for these Borel sums: Namely, $\mathcal{S}_{+}\left[e^{W_{\beta}}\right] \neq \mathcal{S}_{-}\left[e^{W_{\beta}}\right]$ etc. may hold as analytic functions on $\{\eta \in \mathbb{R} \mid \eta \gg 1\}$ (c.f., Vor83, DDP93).

For the mutation of Stokes graphs relevant to a Stokes segment of type $I$ and type III, the jump formulas describing the relationships between these Borel sums 


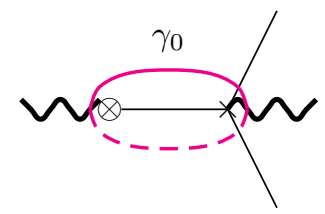

Figure 7. The cycle $\gamma_{0}$ associated with a Stokes segment of type II. The solid part of $\gamma_{0}$ lies on the first sheet of $\hat{\Sigma}$, while the dotted part of $\gamma_{0}$ lies on the second sheet of $\hat{\Sigma}$.

of the Voros symbols are known (see DDP93, AIT]). We find the following formula for a Stokes segment of type II, which is the first main result of this paper.

Theorem 2.17. Assume that the Stokes graph has a unique Stokes segment $\ell_{0}$, which is of type II, connecting a turning point and a simple pole s. Then, the limits of Borel sums (2.31) of the Voros symbols for any path $\beta$ and any cycle $\gamma$ satisfy the following relations as analytic functions of $\eta$ on $\{\eta \in \mathbb{R} \mid \eta \gg 1\}$ :

$$
\left\{\begin{array}{l}
\mathcal{S}_{-}\left[e^{W_{\beta}}\right]=\mathcal{S}_{+}\left[e^{W_{\beta}}\right]\left(1+\left(t+t^{-1}\right) \mathcal{S}_{+}\left[e^{V_{\gamma_{0}}}\right]+\mathcal{S}_{+}\left[e^{2 V_{\gamma_{0}}}\right]\right)^{-\left\langle\gamma_{0}, \beta\right\rangle} \\
\mathcal{S}_{-}\left[e^{V_{\gamma}}\right]=\mathcal{S}_{+}\left[e^{V_{\gamma}}\right]\left(1+\left(t+t^{-1}\right) \mathcal{S}_{+}\left[e^{V_{\gamma_{0}}}\right]+\mathcal{S}_{+}\left[e^{2 V_{\gamma_{0}}}\right]\right)^{-\left(\gamma_{0}, \gamma\right)}
\end{array}\right.
$$

Here

- $t=t(s)$ is given by (2.22),

- $\gamma_{0} \in H_{1}(\hat{\Sigma} \backslash \hat{P})$ is a cycle which surrounds the Stokes segment $\ell_{0}$ (see Figure 7) whose orientation is given by the condition

$$
\oint_{\gamma_{0}} \sqrt{Q_{0}(z)} d z \in \mathbb{R}_{<0}
$$

- $\langle$,$\rangle is the intersection form on the homology groups:$

$$
\langle,\rangle: H_{1}(\hat{\Sigma} \backslash \hat{P}) \times H_{1}\left(\hat{\Sigma} \backslash \hat{P}_{0}, \hat{P}_{\infty}\right) \rightarrow \mathbb{Z},
$$

normalized as

$$
\langle x \text {-axis, } y \text {-axis }\rangle=+1
$$

- $\left(\right.$, ) is the restriction of $\langle$,$\rangle on H_{1}(\hat{\Sigma} \backslash \hat{P}) \times H_{1}(\hat{\Sigma} \backslash \hat{P})$.

Theorem 2.17 is proved in next subsection.

Remark 2.18. Koike and Takei KT11 has already derived the same formula for the Whittaker equation, which is a particular example having a Stokes segment of type II. Theorem 2.17 generalizes the result of [KT11] to the general case. Kamimoto et al. KKKT10 also analyze the singularity structure of Borel transform of the WKB solutions (via a "WKB theoretic transformation") when a Stokes segment of type II appears. Theorem 2.17 follows from the result of KKKT10 if their formal transformation series is Borel summable (see [KK11]).

Remark 2.19. The jump (2.32) for Voros symbols can be interpreted as the Stokes phenomenon (in $\eta \rightarrow+\infty$ ) for the Voros symbols since the both $\mathcal{S}_{+}\left[e^{W_{\beta}}\right]$ and $\mathcal{S}_{-}\left[e^{W_{\beta}}\right]$ has $e^{W_{\beta}}$ as the asymptotic expansion for $\eta \rightarrow+\infty$. Note that, not all 


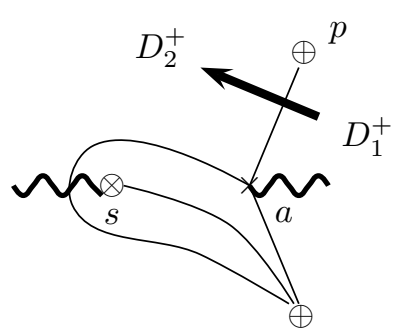

(A) $: G_{+\delta}$

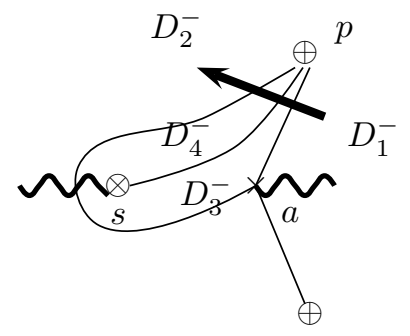

(B) $: G_{-\delta}$

FiguRE 8. Two connection problems.

Voros symbols jump; if a path $\beta$ or a cycle $\gamma$ never intersects with the Stokes segment $\ell_{0}$, the jump formula becomes trivial (i.e., $\mathcal{S}_{-}\left[e^{W_{\beta}}\right]=\mathcal{S}_{+}\left[e^{W_{\beta}}\right]$ etc.) since the intersection number of such paths or cycles and $\gamma_{0}$ is equal to 0 .

2.8. Proof of Theorem 2.17. The strategy of the proof is similar to the one for a type I or type III Stokes segment presented in [IN14, Appendix A, B]. Let us consider two connection problems for certain WKB solutions indicated in Figure 8. The first one (A) is the connection problem from the Stokes region $D_{1}^{+}$to $D_{2}^{+}$ in the Stokes graph $G_{+\delta}$, while the second one (B) is the connection problem from the Stokes region $D_{1}^{-}$to $D_{2}^{-}$(through the intermediate Stokes regions $D_{3}^{-}$and $D_{4}^{-}$) in the Stokes graph $G_{-\delta}$ along the thick paths depicted in Figure 8 Here, after taking a branch cut as in Figure 8, we have specified the branch of $\sqrt{Q_{0}(z)}$ so that the signs of Stokes curves are assigned as in Figure 8 , where the sign is defined as follows. To a Stokes curve emanating from $b \in P_{0} \cup P_{\mathrm{S}}$ in the Stokes graph $G_{ \pm \delta}$, we assign $\oplus$ (resp., $\ominus$ ) if the sign of $\operatorname{Re}\left(e^{ \pm i \delta} \int_{b}^{z} \sqrt{Q_{0}(z)} d z\right)$ on the Stokes curve near its end-point is positive (resp., negative). We can show the desired formula (2.32) in the same manner as presented here if the signs are assigned in the opposite way. We also note that, since the Stokes graph $G_{ \pm \delta}$ is saddle-free, the point $p$ in Figure 8. which is the end-point of a Stokes curve emanating from $a$, is a point in $P_{\infty}$.

If $\psi_{ \pm}(z, \eta)$ are the WKB solutions of (2.1), then

$$
\psi_{ \pm}^{(\theta)}(z, \eta)=\psi_{ \pm}\left(z, e^{i \theta} \eta\right)
$$

are the WKB solutions for $S^{1}$-family of Schödinger equations with potential (2.27). Take the WKB solutions

$$
\psi_{ \pm, a}(z, \eta)=\frac{1}{\sqrt{S_{\text {odd }}(z, \eta)}} \exp \left( \pm \int_{a}^{z} S_{\text {odd }}(z, \eta) d z\right)
$$

of (2.1), which is normalized at the turning point $a$ depicted in Figure 8 , We will compare the two connection formulas for $\psi_{ \pm, a}^{(+\delta)}$ (in Figure $8(\mathrm{~A})$ ) and for $\psi_{ \pm, a}^{(-\delta)}$ (in Figure 8 (B)).

We denote by $\Psi_{ \pm, a}^{(\theta), D}$ the Borel sum of $\psi_{ \pm, a}^{(\theta)}(z, \eta)$ in a Stokes region $D$. Using Theorem 2.12 we have the following formula for the first connection problem (A).

$$
(\mathrm{A}):\left\{\begin{array}{l}
\Psi_{+, a}^{(+\delta), D_{1}^{+}}=\Psi_{+, a}^{(+\delta), D_{2}^{+}}+i \Psi_{-, a}^{(+\delta), D_{2}^{+}} \\
\Psi_{-, a}^{(+\delta), D_{1}^{+}}=\Psi_{-, a}^{(+\delta), D_{2}^{+}}
\end{array}\right.
$$


On the other hand, in the second connection problem (B) we have to cross three Stokes curves emanating from $a$ and $s$ as in Figure 8 . The resulting connection formula is given as follows.

Lemma 2.20. In the second connection problem (B) we have

$$
(\mathrm{B}):\left\{\begin{array}{l}
\Psi_{+, a}^{(-\delta), D_{1}^{-}}=\Psi_{+, a}^{(-\delta), D_{2}^{-}}+i\left(1+\left(t+t^{-1}\right) \mathcal{S}\left[e^{V_{\gamma_{0}}^{(-\delta)}}\right]+\mathcal{S}\left[e^{2 V_{\gamma_{0}}^{(-\delta)}}\right]\right) \Psi_{-, a}^{(-\delta), D_{2}^{-}} \\
\Psi_{-, a}^{(-\delta), D_{1}^{-}}=\Psi_{-, a}^{(-\delta), D_{2}^{-}}
\end{array}\right.
$$

Proof. Let us describe the connection formulas for WKB solutions near the three crossing points.

- Connection formula for $D_{1}^{-} \rightsquigarrow D_{3}^{-}$. Using Theorem 2.12 again, we have

$$
\left\{\begin{array}{l}
\Psi_{+, a}^{(-\delta), D_{1}^{-}}=\Psi_{+, a}^{(-\delta), D_{3}^{-}}+i \Psi_{-, a}^{(-\delta), D_{3}^{-}} \\
\Psi_{-, a}^{(-\delta), D_{1}^{-}}=\Psi_{-, a}^{(-\delta), D_{3}^{-}}
\end{array}\right.
$$

- Connection formula for $D_{3}^{-} \rightsquigarrow D_{4}^{-}$. Here we need to use Theorem 2.13 which describes the connection formula on a Stokes curve emanating from the simple pole $s$. In order to use Theorem 2.13, we need to change the normalization of the WKB solutions since Theorem 2.13 is only valid for the WKB solutions normalized at a simple pole. For the WKB solution

$$
\psi_{ \pm, s}(z, \eta)=\frac{1}{\sqrt{S_{\text {odd }}(z, \eta)}} \exp \left( \pm \int_{s}^{z} S_{\text {odd }}(z, \eta) d z\right)
$$

of (2.1) normalized at $s$, the following formula holds on the Stokes curve in question:

$$
\left\{\begin{array}{l}
\Psi_{+, s}^{(-\delta), D_{3}^{-}}=\Psi_{+, s}^{(-\delta), D_{4}^{-}}+i\left(t+t^{-1}\right) \Psi_{-, s}^{(-\delta), D_{4}^{-}} \\
\Psi_{-, s}^{(-\delta), D_{3}^{-}}=\Psi_{-, s}^{(-\delta), D_{4}^{-}}
\end{array}\right.
$$

Here $\Psi_{ \pm, s}^{(-\delta), D_{3}^{-}}$etc. are the Borel sum of $\psi_{ \pm, s}^{(-\delta)}(z, \eta)$ in the Stokes region $D_{3}^{-}$etc. Then, using the relation

$$
\psi_{ \pm, a}^{(\theta)}(z, \eta)=\exp \left( \pm \int_{a}^{s} S_{\text {odd }}\left(z, e^{i \theta} \eta\right) d z\right) \psi_{ \pm, s}^{(\theta)}(z, \eta)=e^{ \pm \frac{1}{2} V_{\gamma_{0}}^{(\theta)}(\eta)} \psi_{ \pm, s}^{(\theta)}(z, \eta)
$$

(here the cycle $\gamma_{0}$ satisfying (2.33) has the orientation as depicted in Figure 9 (a), and we have used the equality (2.30) $)$, the formula (2.42) is translated to a formula for the Borel sum of the WKB solutions $\psi_{ \pm, a}^{(-\delta)}$ as follows:

$$
\left\{\begin{array}{l}
\Psi_{+, a}^{(-\delta), D_{3}^{-}}=\Psi_{+, a}^{(-\delta), D_{4}^{-}}+i\left(t+t^{-1}\right) \mathcal{S}\left[e^{V_{\gamma_{0}}^{(-\delta)}}\right] \Psi_{-, a}^{(-\delta), D_{4}^{-}} \\
\Psi_{-, a}^{(-\delta), D_{3}^{-}}=\Psi_{-, a}^{(-\delta), D_{4}^{-}} .
\end{array}\right.
$$

- Connection formula for $D_{4}^{-} \rightsquigarrow D_{2}^{-}$. Let us introduce another WKB solutions

$$
\varphi_{ \pm, a}(z, \eta)=\frac{1}{\sqrt{S_{\text {odd }}(z, \eta)}} \exp \left( \pm \int_{a}^{z} S_{\text {odd }}(z, \eta) d z\right)
$$

where the path of integration is taken along the path $\gamma_{z}^{(2)}$ depicted in Figure 9 (b). (The path for the original WKB solutions $\psi_{ \pm, a}(z, \eta)$ is taken along the path $\gamma_{z}^{(1)}$ 


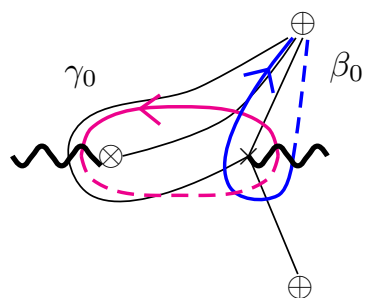

(a) The cycle $\gamma_{0}$ and the path $\beta_{0}$.

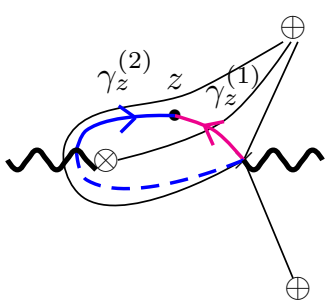

(b) The path $\gamma_{z}^{(1)}$ and $\gamma_{z}^{(2)}$

Figure 9. The paths and cycles.

in Figure 9 (b).) Then, Theorem 2.12 is valid for the Borel sum of $\varphi_{ \pm, a}^{(-\delta)}$ on the Stokes curve in question (see Section 2.5):

$$
\left\{\begin{array}{l}
\Phi_{+, a}^{(-\delta), D_{4}^{-}}=\Phi_{+, a}^{(-\delta), D_{2}^{-}}+i \Phi_{-, a}^{(-\delta), D_{2}^{-}} \\
\Phi_{-, a}^{(-\delta), D_{4}^{-}}=\Phi_{-, a}^{(-\delta), D_{2}^{-}}
\end{array}\right.
$$

Here $\Phi_{ \pm, a}^{(-\delta), D_{4}^{-}}$etc. are the Borel sum of $\varphi_{ \pm, a}^{(-\delta)}(z, \eta)$ in the Stokes region $D_{4}^{-}$etc. Since $\psi_{ \pm, a}^{(\theta)}$ and $\varphi_{ \pm, a}^{(\theta)}$ are related as

$$
\psi_{ \pm, a}^{(\theta)}(z, \eta)=e^{ \pm V_{\gamma_{0}}^{(\theta)}(\eta)} \varphi_{ \pm, a}^{(\theta)}(z, \eta),
$$

we obtain the following formula for the Borel sum of the original WKB solutions $\psi_{ \pm, a}^{(-\delta)}$ from (2.46):

$$
\left\{\begin{array}{l}
\Psi_{+, a}^{(-\delta), D_{4}^{-}}=\Psi_{+, a}^{(-\delta), D_{2}^{-}}+i \mathcal{S}\left[e^{2 V_{\gamma_{0}}^{(-\delta)}}\right] \Psi_{-, a}^{(-\delta), D_{2}^{-}} \\
\Psi_{-, a}^{(-\delta), D_{4}^{-}}=\Psi_{-, a}^{(-\delta), D_{2}^{-}} .
\end{array}\right.
$$

Therefore, combining (2.40), (2.44) and (2.48), we have the connection formula (2.39) for the second connection problem (B).

Next, let us rewrite the formulas (2.38) and (2.39) to the formulas for the WKB solutions

$$
\psi_{ \pm, p}(z, \eta)=\frac{1}{\sqrt{S_{\text {odd }}(z, \eta)}} \exp \left\{ \pm\left(\eta \int_{a}^{z} \sqrt{Q_{0}(z)} d z+\int_{p}^{z} S_{\text {odd }}^{\text {reg }}(z, \eta) d z\right)\right\}
$$

of (2.1) normalized at $p \in P_{\infty}$, which is an end-point of a Stokes curve emanating from $a$ as depicted in Figure 8 . The WKB solutions $\psi_{ \pm, a}^{(\theta)}$ and $\psi_{ \pm, p}^{(\theta)}$ are related as

$$
\psi_{ \pm, a}^{(\theta)}(z, \eta)=\exp \left( \pm \int_{a}^{p} S_{\mathrm{odd}}^{\mathrm{reg}}\left(z, e^{i \theta} \eta\right) d z\right) \psi_{ \pm, p}^{(\theta)}=e^{ \pm \frac{1}{2} W_{\beta_{0}}^{(\theta)}(\eta)} \psi_{ \pm, p}^{(\theta)}(z, \eta)
$$


where $\beta_{0}$ is the path designated in Figure 9 (a). Therefore, thanks to (2.50), (2.38) and (2.39), we obtain the following equalities:

$$
(\mathrm{A}):\left\{\begin{array}{l}
\Psi_{+, p}^{(+\delta), D_{1}^{+}}=\Psi_{+, p}^{(+\delta), D_{2}^{+}}+i \mathcal{S}\left[e^{-W_{\beta_{0}}^{(+\delta)}}\right] \Psi_{-, p}^{(+\delta), D_{2}^{+}} \\
\Psi_{-, p}^{(+\delta), D_{1}^{+}}=\Psi_{-, p}^{(+\delta), D_{2}^{+}} .
\end{array}\right.
$$

$$
(\mathrm{B}):\left\{\begin{array}{l}
\Psi_{+, p}^{(-\delta), D_{1}^{-}}=\Psi_{+, p}^{(-\delta), D_{2}^{-}} \\
\quad+i\left(1+\left(t+t^{-1}\right) \mathcal{S}\left[e^{V_{\gamma_{0}}^{(-\delta)}}\right]+\mathcal{S}\left[e^{2 V_{\gamma_{0}}^{(-\delta)}}\right]\right) \mathcal{S}\left[e^{-W_{\beta_{0}}^{(-\delta)}}\right] \Psi_{-, p}^{(-\delta), D_{2}^{-}} \\
\Psi_{-, p}^{(-\delta), D_{1}^{-}}=\Psi_{-, p}^{(-\delta), D_{2}^{-}} .
\end{array}\right.
$$

Taking $\delta>0$ sufficiently small, we may assume that, for a fixed $z_{1} \in D_{1}^{+} \cap D_{1}^{-}$ (resp., $z_{2} \in D_{2}^{+} \cap D_{2}^{-}$) the path from $p$ to $z$, which normalizes the WKB solution (2.49) when $z$ lies in a neighborhood of $z_{1}$ (resp., $z_{2}$ ), never intersects with the Stokes curves in $G_{\theta}$ for any $\theta$ satisfying $-\delta \leq \theta \leq+\delta$. Therefore, Theorem 2.10 implies that $\psi_{ \pm, p}^{(\theta)}$ is Borel summable for any $\theta$ satisfying $-\delta \leq \theta \leq+\delta$. Then, the same discussion in the proof of [IN14, Proposition 2.23, Lemma 3.9] shows that

$$
\lim _{\delta \rightarrow+0} \Psi_{ \pm, p}^{(+\delta), D_{1}^{+}}=\lim _{\delta \rightarrow+0} \Psi_{ \pm, p}^{(-\delta), D_{1}^{-}}
$$

holds as analytic functions of both $z$ (near $z_{1}$ ) and $\eta$ (for a sufficiently large $\eta>0$ ). Similarly,

$$
\lim _{\delta \rightarrow+0} \Psi_{ \pm, p}^{(+\delta), D_{2}^{+}}=\lim _{\delta \rightarrow+0} \Psi_{ \pm, p}^{(-\delta), D_{2}^{-}}
$$

also holds when $z$ lies near $z_{2}$. Therefore, comparing the connection multipliers in (2.51) and (2.52) after taking the limit $\delta \rightarrow+0$, we obtain

$$
\mathcal{S}_{-}\left[e^{W_{\beta_{0}}}\right]=\mathcal{S}_{+}\left[e^{W_{\beta_{0}}}\right]\left(1+\left(t+t^{-1}\right) \mathcal{S}_{+}\left[e^{V_{\gamma_{0}}}\right]+\mathcal{S}_{+}\left[e^{2 V_{\gamma_{0}}}\right]\right) .
$$

Here we have used the equality

$$
\mathcal{S}_{-}\left[e^{V_{\gamma_{0}}}\right]=\mathcal{S}_{+}\left[e^{V_{\gamma_{0}}}\right],
$$

which follows from the fact that $\gamma_{0}$ does not intersect with the Stokes segment $\ell_{0}$ (see [IN14, Proposition 2.23] and Remark 2.19). The equality (2.55) is the desired formula (2.32) for the path $\beta_{0}$. (Note that $\left\langle\gamma_{0}, \beta_{0}\right\rangle=-1$.) The formula (2.32) for a general path $\beta$ and a general cycle $\gamma$ can be derived from (2.55) by the same manner as in the proof of [IN14, Theorem 3.4 (Appendix A)]. This completes the proof of Theorem 2.17

Remark 2.21. Since the WKB solutions $\psi_{ \pm, a}$ defined by (2.37) are normalized along a path which intersects with the Stokes segment $\ell_{0}$, we cannot expect similar equalities as (2.53) and (2.54) hold for the Borel sums of $\psi_{ \pm, a}$.

In our previous paper IN14, we found that the jump formulas for Stokes segments of type I and type III obtained in [DDP93, AIT can be formulated as a (signed) mutation in cluster algebras. We will see that the jump formula for Voros symbols for a type II Stokes segment shown in Theorem 2.17 is recognized as a (signed) mutation in generalized cluster algebras in the sense of [CS14 in Section 4 


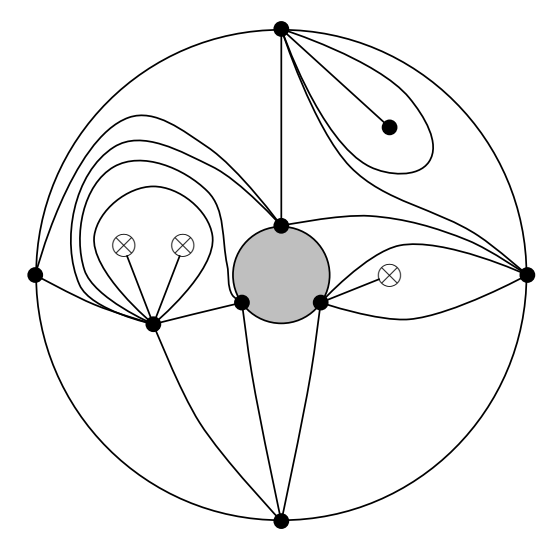

FiguRE 10. Example of an ideal triangulation of an annulus with two punctures and three orbifold points.

\section{Orbifold triangulations And Stokes graphs}

3.1. Ideal triangulations of orbifolds. In this subsection we recall some basic notions and properties of ideal triangulations of orbifolds introduced in [FST11]. Here we concentrate on the orbifold points of weight 2 therein.

Definition 3.1. Let $\mathbf{S}$ be any compact connected oriented surface possibly with boundary. Let $\mathbf{M}$ be a finite set of marked points on $\mathbf{S}$ that is nonempty and includes at least one marked point on each boundary component of $\mathbf{S}$ and possibly some interior points of $\mathbf{S}$. Let $\mathbf{Q}$ be a finite nonempty set of interior points of $\mathbf{S}$ such that $\mathbf{M} \cap \mathbf{Q}=\emptyset$. The triplet $\mathbf{O}=(\mathbf{S}, \mathbf{M}, \mathbf{Q})$ is called a bordered orbifold with marked points, or simply, an orbifold. An interior point of $\mathbf{M}$ is called a puncture. A point of $\mathbf{Q}$ is called an orbifold point. In figures, a marked point and an orbifold point is shown by $\bullet$ and $\otimes$, respectively. See Figure 10 for an example.

Definition 3.2. An arc $\alpha$ in an orbifold $\mathbf{O}$ is a curve in $\mathbf{S}$ such that

- one of the following holds:

- both endpoints of $\alpha$ are marked points (then $\alpha$ is called an ordinary arc),

- one end point of $\alpha$ is a marked point and the other end point is an orbifold point (then $\alpha$ is called a pending arc),

- $\alpha$ does not intersect itself except for the endpoints,

- $\alpha$ is away from punctures, orbifold points, and the boundary except for the endpoints,

- $\alpha$ is not contractible into a marked point or onto a boundary of $\mathbf{S}$,

- $\alpha$ does not cut out a monogon containing exactly one orbifold point.

Furthermore, each arc is considered up to isotopy in the class of such curves.

Definition 3.3. Two arcs are said to be compatible if

- there are representatives in their respective isotopy classes such that they do not intersect each other in the interior of $\mathbf{S}$,

- they do not share an orbifold point as a common end point. 


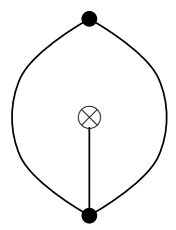

type i

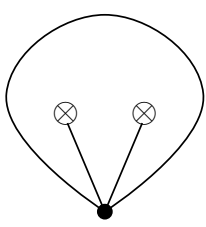

type ii

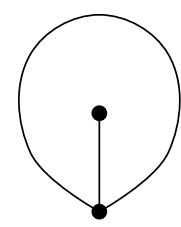

type iii

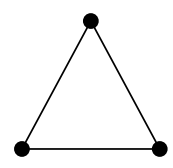

type iv

Figure 11. Pieces of labeled ideal triangulations of orbifolds.

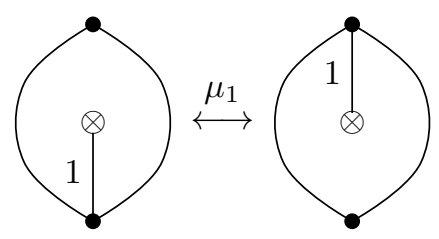

FiguRE 12. Flips of pending arcs in type i pieces.

Definition 3.4. A labeled ideal triangulation $T$ of an orbifold $\mathbf{O}$ is an $n$-tuple of $\operatorname{arcs}\left(\alpha_{i}\right)_{i=1}^{n}$ in $\mathbf{O}$ which gives a maximal set of distinct pairwise compatible arcs.

Caution 3.5. This should not be confused with a tagged triangulation of an orbifold also studied in [FST11. We do not use tagged triangulations in this paper.

Figure 10 shows an example of a labeled ideal triangulation of an annulus with two punctures and three orbifold points, where the labels of arcs are omitted for simplicity.

It was explained in FST11 that any labeled ideal triangulation of an orbifold is built by glueing the edges of four kinds of "puzzle pieces" depicted in Figure 11] We call them type $i$, type ii, type iii, and type iv pieces, respectively, as specified in Figure 11. We remark that, there are actually infinitely many distinct type ii pieces of given two orbifold points and a puncture. See Figure 13. Note that orbifold points and pending arcs are always inside type $\mathrm{i}$ or type ii pieces.

Definition 3.6. For a given labeled ideal triangulation $T=\left(\alpha_{i}\right)_{i=1}^{n}$ of an orbifold $\mathbf{O}$, a flip of $T$ at $k(k=1, \ldots, n)$ is another labeled ideal triangulation $T^{\prime}=\left(\alpha_{i}^{\prime}\right)_{i=1}^{n}$ of $\mathbf{O}$ such that $\alpha_{i}^{\prime}=\alpha_{i}$ for $i \neq k$ and $\alpha_{k}^{\prime} \neq \alpha_{k}$.

Lemma 3.7 ([FST08, FST11]). For a given triangulation $T=\left(\alpha_{i}\right)_{i=1}^{n}$ of an orbifold $\mathbf{O}$, the flip $T^{\prime}=\mu_{k}(T)$ exists if and only if the arc $\alpha_{k}$ is not inside a type iii piece of $T$; moreover, under the same condition $T^{\prime}$ exists uniquely. In particular, any pending arcs can be flipped.

Note that $T^{\prime}=\mu_{k}(T)$ if and only if $T=\mu_{k}\left(T^{\prime}\right)$. Namely, $\mu_{k}$ is an involution.

Example 3.8. Flips of pending arcs are shown in Figures 12 and 13 .

The following property is a consequence of the transitivity of flips on (unlabeled) ideal triangulations of an orbifold given by [FST11, Theorem 4.2].

Proposition 3.9 ([FST11, Theorem 4.2]). Let $\mathbf{O}$ be an orbifold, and let $T=$ $\left(\alpha_{i}\right)_{i=1}^{n}$ be a labeled ideal triangulation of $\mathbf{O}$. Then, the total number $(=n)$ of arcs of $T$ and the total number of pieces in $T$ do not depend on $T$. 


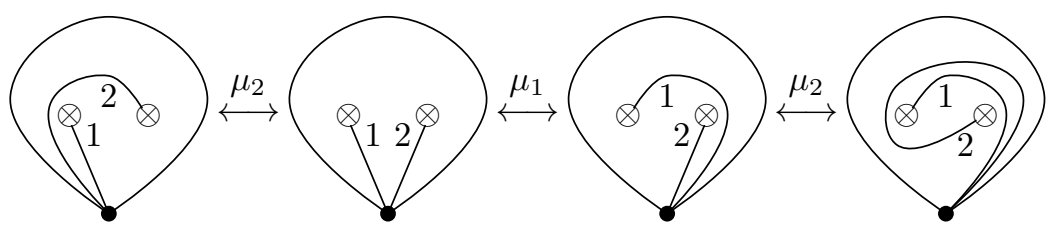

Figure 13. Flips of pending arcs in type ii pieces.

3.2. Signed adjacency matrix. To each labeled ideal triangulation $T=\left(\alpha_{i}\right)_{i=1}^{n}$ of an orbifold, we attach a skew-symmetrizable integer matrix $B=B(T)=$ $\left(b_{i j}\right)_{i, j=1}^{n}$ called the signed adjacency matrix of $T$ (see [FST08, Section 4] and [FST11, Table 3.4]). In this paper we do not need its full definition, which is somewhat cumbersome to describe, and only the submatrices of $B$ corresponding to type i and type ii pieces of $T$ are essential. They are explicitly given as follows:

Type i piece:

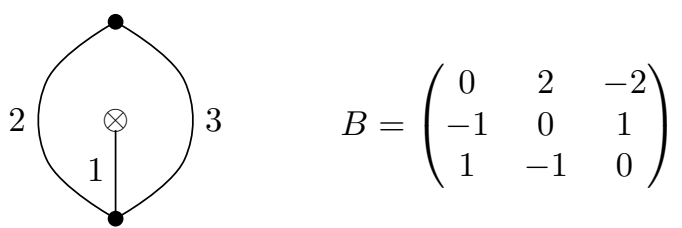

Type ii piece: The matrix $B$ depends only on the relative position of arcs near the marked point, and it is independent of how they reach to orbifold points.

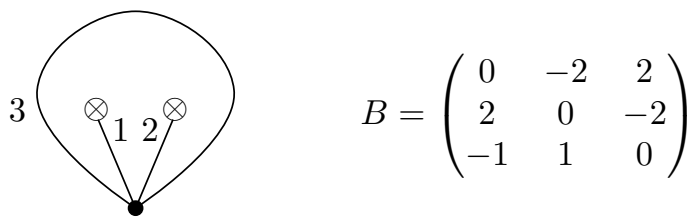

We set the weight $d_{i}$ of the $\operatorname{arc} \alpha_{i}$ in $T$ as

$$
d_{i}= \begin{cases}1 & \alpha_{i} \text { is an ordinary arc } \\ 2 & \alpha_{i} \text { is a pending arc. }\end{cases}
$$

Introduce the diagonal matrix $D=\left(d_{i} \delta_{i j}\right)_{i, j=1}^{n}$. Then, $B D$ and $\tilde{B}=D^{-1} B$ are skew-symmetric and integer matrices. For example, in the above two cases, we have respectively

$$
\begin{aligned}
& B D=\left(\begin{array}{ccc}
0 & 2 & -2 \\
-2 & 0 & 1 \\
2 & -1 & 0
\end{array}\right), \quad \tilde{B}=D^{-1} B=\left(\begin{array}{ccc}
0 & 1 & -1 \\
-1 & 0 & 1 \\
1 & -1 & 0
\end{array}\right), \\
& B D=\left(\begin{array}{ccc}
0 & -4 & 2 \\
4 & 0 & -2 \\
-2 & 2 & 0
\end{array}\right), \quad \tilde{B}=D^{-1} B=\left(\begin{array}{ccc}
0 & -1 & 1 \\
1 & 0 & -1 \\
-1 & 1 & 0
\end{array}\right) \text {. }
\end{aligned}
$$

The following is the most basic notion in cluster algebra theory [FZ03].

Definition 3.10. For a skew-symmetrizable matrix $B=\left(b_{i j}\right)_{i, j=1}^{n}$, the mutation of $B$ at $k(k=1, \ldots, n)$ is another skew-symmetrizable matrix $B^{\prime}=\mu_{k}(B)=\left(b_{i j}^{\prime}\right)_{i, j=1}^{n}$ 
defined by the following relation:

$$
b_{i j}^{\prime}= \begin{cases}-b_{i j} & i=k \text { or } j=k \\ b_{i j}+\left[-b_{i k}\right]_{+} b_{k j}+b_{i k}\left[b_{k j}\right]_{+} & i, j \neq k\end{cases}
$$

where $[a]_{+}=\max (a, 0)$.

The flips of labeled ideal triangulations of an orbifold and the mutations of their signed adjacency matrices are compatible in the following sense.

Proposition 3.11 ([FST11, Theorem 4.19]). For any labeled ideal triangulation $T=\left(\alpha_{i}\right)_{i=1}^{n}$ of an orbifold $\mathbf{O}$ and any $k$ such that the arc $\alpha_{k}$ is not inside a type iii piece of $T$, we have $B\left(\mu_{k}(T)\right)=\mu_{k}(B(T))$.

Example 3.12. For the matrix $B$ in (3.1), $\mu_{1}(B)=-B$. For the matrix $B$ in (3.2), $\mu_{1}(B)=\mu_{2}(B)=-B$. They certainly agree with Proposition 3.11.

3.3. Stokes triangulations of orbifolds and signed flips of pending arcs. In IN14 the notion of a Stokes triangulation of a bordered surface was introduced. It is a refinement of an ideal triangulation by [FST08] so as to capture the characteristics of the geometry of Stokes graphs especially under the mutation. See also Qiu14. Here we extend it to the orbifold case straightforwardly.

Definition 3.13. For an orbifold $\mathbf{O}=(\mathbf{S}, \mathbf{M}, \mathbf{Q})$, let $m$ be the total number of pieces in any labeled ideal triangulation $T$ of $\mathbf{O}$, where $m$ is independent of the choice of $T$ by Proposition 3.9. Accordingly, we introduce a set $\mathbf{A}$ consisting of $m$ internal points of $\mathbf{O}$ such that $\mathbf{A} \cap \mathbf{M}=\mathbf{A} \cap \mathbf{Q}=\emptyset$. We call $a \in \mathbf{A}$ a midpoint (of a piece), and in figures it will be shown by $\times$. For brevity, we still call $(\mathbf{O}, \mathbf{A})=(\mathbf{S}, \mathbf{M}, \mathbf{Q}, \mathbf{A})$ an orbifold.

Definition 3.14. An arc $\alpha$ in an orbifold $(\mathbf{O}, \mathbf{A})$ is a curve in $\mathbf{S} \backslash \mathbf{A}$ satisfying all conditions in Definition 3.2. Each arc $\alpha$ is considered up to isotopy in the class of such curves.

When we consider an $\operatorname{arc} \alpha$ in $(\mathbf{O}, \mathbf{A})$, it is sometimes convenient to regard it as an $\operatorname{arc}$ in $\mathbf{O}$ by forgetting the midpoints. In that case we write the latter arc as $\tilde{\alpha}$ to avoid confusion.

Definition 3.15. An $n$-tuple $T=\left(\alpha_{i}\right)_{i=1}^{n}$ of $\operatorname{arcs}$ in $(\mathbf{O}, \mathbf{A})$ is called a labeled Stokes triangulation of $(\mathbf{O}, \mathbf{A})$ if the following conditions are satisfied:

- The $\operatorname{arcs} \alpha_{1}, \ldots, \alpha_{n}$ in $(\mathbf{O}, \mathbf{A})$ are pairwise compatible (in the sense of Definition 3.3 but considered in the isotopy classes for arcs in $(\mathbf{O}, \mathbf{A}))$.

- The $n$-tuple $\tilde{T}=\left(\tilde{\alpha}_{i}\right)_{i=1}^{n}$ of $\operatorname{arcs}$ in $\mathbf{O}$ yields a labeled ideal triangulation of O.

- Every piece of $T$ contains exactly one midpoint in its interior. Here, a piece of $T$ is a collection of $\operatorname{arcs}$ of $T$ such that it yields a piece of $\tilde{T}$ as arcs in $\mathbf{O}$.

For a labeled Stokes triangulation $T$ of $(\mathbf{O}, \mathbf{A})$, its signed adjacency matrix $B(T)$ and weights of $\operatorname{arcs}$ in $T$ are defined by those of the underlying labeled ideal triangulation $\tilde{T}$.

Again, pieces of a labeled Stokes triangulation $T$ fall into four types (from type $\mathrm{i}$ to type iv) depending on their types as pieces in $\tilde{T}$.

Next we introduce the signed flips for labeled Stokes triangulations. Like the ordinary flip of an arc of a labeled ideal triangulation, they are defined if an arc 


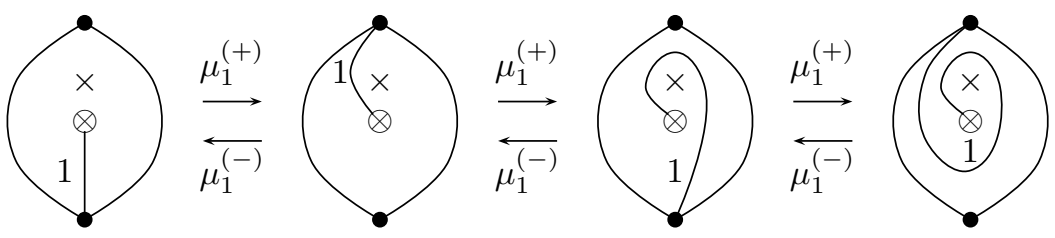

Figure 14. Signed flips of pending arcs in type i pieces.

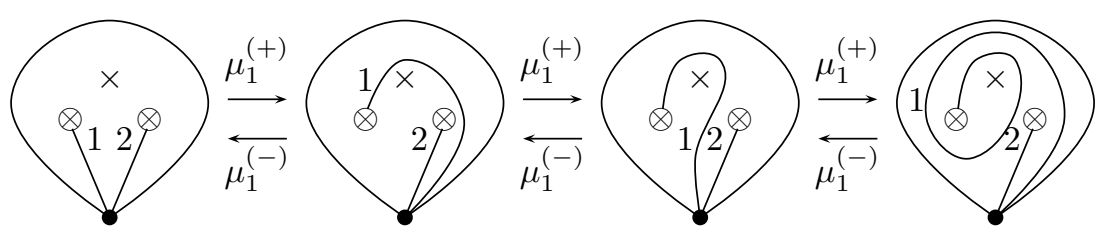

FiguRE 15. Signed flips of pending arcs in type ii pieces.

is not inside a type iii piece of a triangulation. Here we concentrate on the signed flips of pending arcs. See [IN14, Definition 6.4] for the signed flips of ordinary arcs.

Definition 3.16. For a labeled Stokes triangulation $T=\left(\alpha_{i}\right)_{i=1}^{n}$ of an orbifold $(\mathbf{O}, \mathbf{A})$, a pending arc $\alpha_{k}$, and a sign $\varepsilon \in\{+,-\}$, the signed flip $T^{\prime}=\mu_{k}^{(\varepsilon)}(T)$ at $k$ with sign $\varepsilon$ is another labeled Stokes triangulation of $(\mathbf{O}, \mathbf{A})$ obtained from $T$ by replacing the arc $\alpha_{k}$ with the one in Figures 14 and 15. Namely,

- if the pending arc $\alpha_{k}$ is in a type $i$ piece, $\alpha_{k}^{\prime}$ is obtained from $\alpha_{k}$ by sliding its outside end point from a marked point to the other marked point along the boundary of the piece clockwise for $\varepsilon=+$ and anticlockwise for $\varepsilon=-$,

- if the pending arc $\alpha_{k}$ is in a type ii piece, $\alpha_{k}^{\prime}$ is obtained from $\alpha_{k}$ by sliding its outside end point from a marked point to itself along the boundary or along the other pending arc in the piece clockwise for $\varepsilon=+$ and anticlockwise for $\varepsilon=-$.

The signed flips are compatible with the flip of the underlying labeled ideal triangulation; i.e., for $T^{\prime}=\mu_{k}^{(\varepsilon)}(T)$ with any $\varepsilon= \pm, \tilde{T}=\mu_{k}(\tilde{T})$ holds. In particular, they are also compatible with the mutation of their signed adjacency matrices, namely, $B\left(T^{\prime}\right)=\mu_{k}(B(T))$.

3.4. Stokes graphs and Stokes triangulations. Here we explain how a Stokes triangulation of an orbifold is obtained from a saddle-free Stokes graph of the Schrödinger equation (2.1) with simple poles.

Recall that the principal term of the potential $Q(z, \eta)$ determines a quadratic differential $\phi$ on the Riemann surface $\Sigma$ (see Definition 2.1), and we have assumed that all zeros of $\phi$ are simple (see Assumption 2.3), and there is no recurrent trajectory (see Assumption 2.77). In this subsection we also assume that

(A) the Stokes graph $G(\phi)$ in Definition 2.6 is saddle-free.

Furthermore, let us temporarily assume that

(B) $\phi$ has no simple poles. 


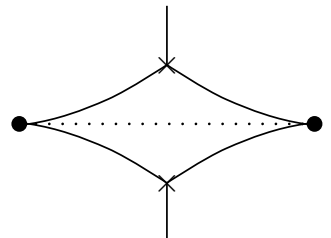

(a)

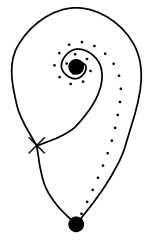

(b)

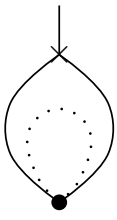

(c)

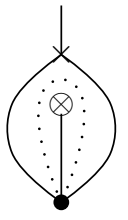

(d)

Figure 16. Patterns of Stokes regions: (a) regular horizontal strip, (b) degenerate horizontal strip, (c) half plane, and (d) horizontal strip of simple-pole type. The dotted lines are representatives of trajectories.

Under such assumptions, any Stokes region of $G(\phi)$ falls into one of three types, i.e., regular horizontal strip, degenerate horizontal strip, or half plane, depicted in Figure 16 (a)-(c) (see BS13, Section 3.5]). The Stokes triangulation $T(\phi)$ of a bordered surface $(\mathbf{S}(\phi), \mathbf{M}(\phi), \mathbf{A}(\phi))$ associated with $G(\phi)$ was introduced in IN14, Section 6], refining the ideas of [KT05, GMN13, BS13. Let us roughly summarize the construction as follows.

- $\mathbf{S}(\phi)$ : This is obtained from $\Sigma$ (as a topological surface) by cutting out small holes around poles of $\phi$ of order greater than 2 .

- $\mathbf{M}(\phi)$ : At each boundary component, $r-2$ marked points are attached, where $r \geq 3$ is the order of the corresponding pole. The poles of order 2 of $\phi$ are the punctures.

- $\mathbf{A}(\phi)$ : The turning points are the midpoints.

- Each arc of $T(\phi)$ corresponds to a representative of trajectories in a horizontal strip. To be more specific, inner arcs in type iii pieces are the ones for degenerate horizontal strips, while the other arcs are for regular horizontal strips.

- Each edge on the boundary of $\mathbf{S}(\phi)$ corresponds to a representative of trajectories in a half plane.

- A label of horizontal strips of $G(\phi)$ gives a label of $T(\phi)$.

Now we remove the condition (B) and allow $\phi$ to have simple poles. Then, we have an additional type of Stokes regions given in Figure 16 (d), which we call a horizontal strip of simple-pole type. Then, in addition to the above, we have the following correspondence shown in Figure 17

- $\mathbf{Q}(\phi)$ : The simple poles of $\phi$ are the orbifold points.

- Each pending arc of $T(\phi)$ corresponds to a representative of trajectories around a simple pole.

As seen in Figure 17, there are two possible arrangements of horizontal strips of simple-pole type in Stokes graphs which correspond to type i and type ii pieces, respectively.

In summary, we obtain the labeled Stokes triangulation $T(\phi)$ of the orbifold $(\mathbf{S}(\phi), \mathbf{M}(\phi), \mathbf{Q}(\phi), \mathbf{A}(\phi))$ which is associated with a saddle-free Stokes graph $G(\phi)$.

3.5. Mutation of Stokes graphs. Now we are at the important stage of combining two main ideas: the mutation of Stokes graphs of the Schrödinger equation (see 

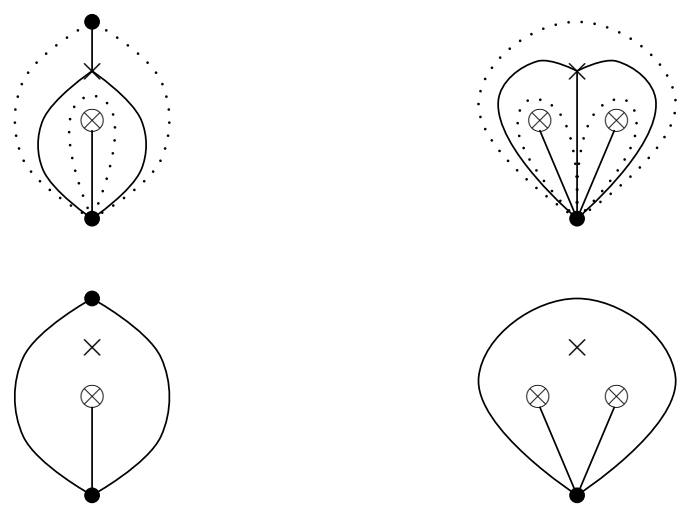

type i

type ii

FiguRE 17. Arrangements of horizontal strips of simple-pole type in Stokes graphs (above) and corresponding Stokes triangulations.

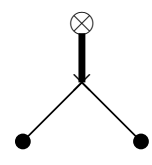

type i-a

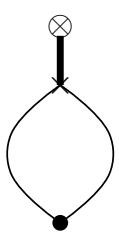

type $\mathrm{i}-\mathrm{b}$

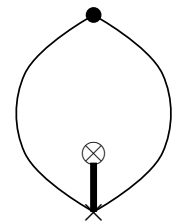

type i-c

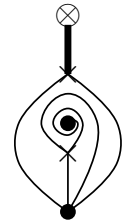

type i-d

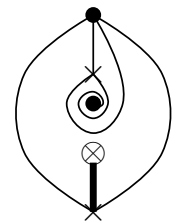

type i-e

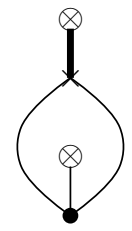

type ii-a

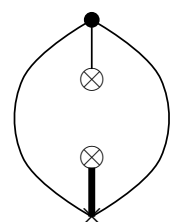

type ii-b

Figure 18. Local configurations near type II Stokes segment. Type II Stokes segments are depicted in bold lines.

Section 2.7) and the signed flips of Stokes triangulations. Here, we concentrate on the situation where the jump formula with respect to a type II Stokes segment in Theorem 2.17 is relevant. See [N14, Section 6.3] for a more general setting.

Let $\phi$ be the quadratic differential associated with the Schrödinger equation (2.1), and $G_{0}=G(\phi)$ be its Stokes graph. We assume that

- $G_{0}$ has a unique Stokes segment, which is of type II.

Then, there are seven possible local configurations near a type II Stokes segment in $G_{0}$ depicted in Figure 18. We call five of them type $i$ configurations and two of them type ii configurations as specified in Figure 18 .

Let $Q^{(\theta)}(z, \eta)$ be the $S^{1}$-family of potentials defined in (2.27). Take a sufficiently small $\delta>0$ and consider the Stokes graphs $G_{ \pm \delta}=G\left(e^{ \pm 2 i \delta} \phi\right)$ for $Q^{( \pm 2 i \delta)}(z, \eta)$. Then, $G_{ \pm \delta}$ are saddle-free as explained in Section 2.7. Let $T_{ \pm \delta}=T\left(e^{ \pm 2 i \delta} \phi\right)$ be the 

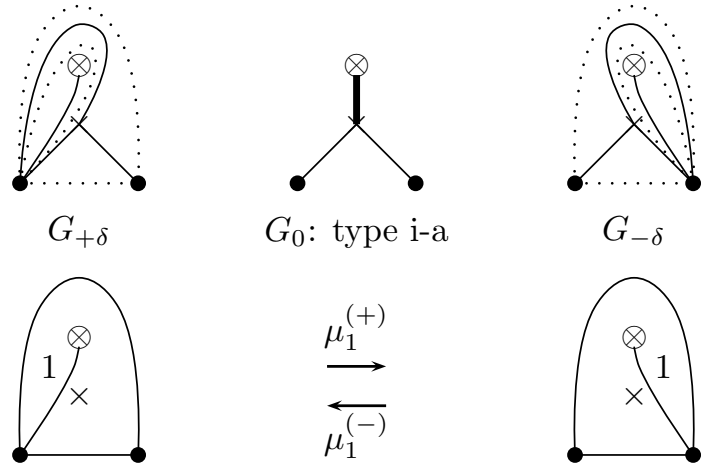

$T_{+\delta}$ : type i

$T_{-\delta}:$ type i
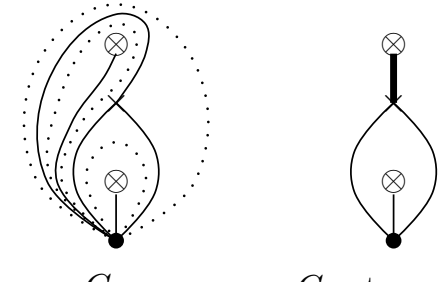

$G_{0}$ : type ii-a
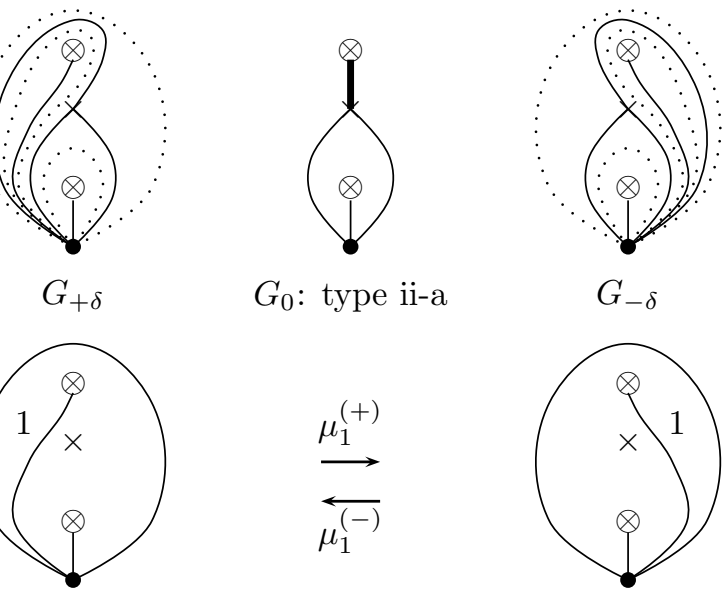

$$
\stackrel{\mu_{1}^{(+)}}{\stackrel{\longleftrightarrow}{\mu_{1}^{(-)}}}
$$

$G_{-\delta}$

$T_{+\delta}$ : type ii
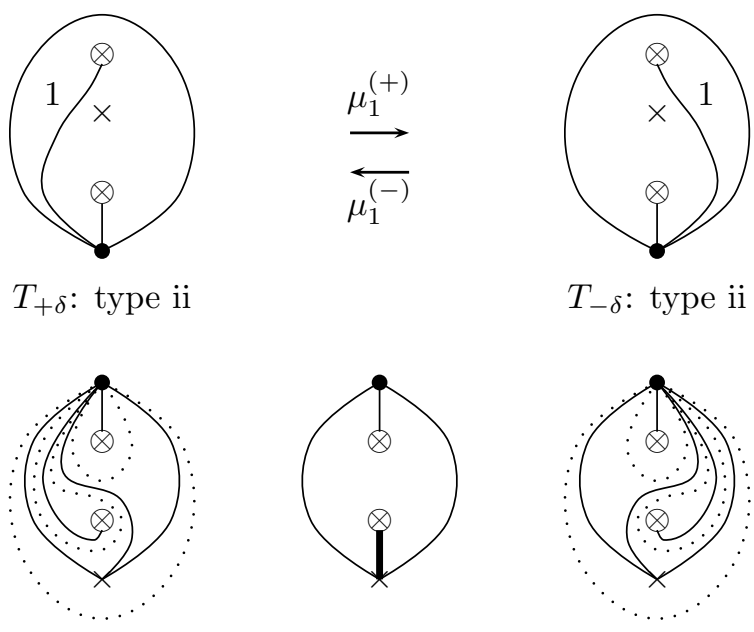

$T_{-\delta}$ : type ii

$G_{+\delta}$

$G_{0}:$ type ii-b
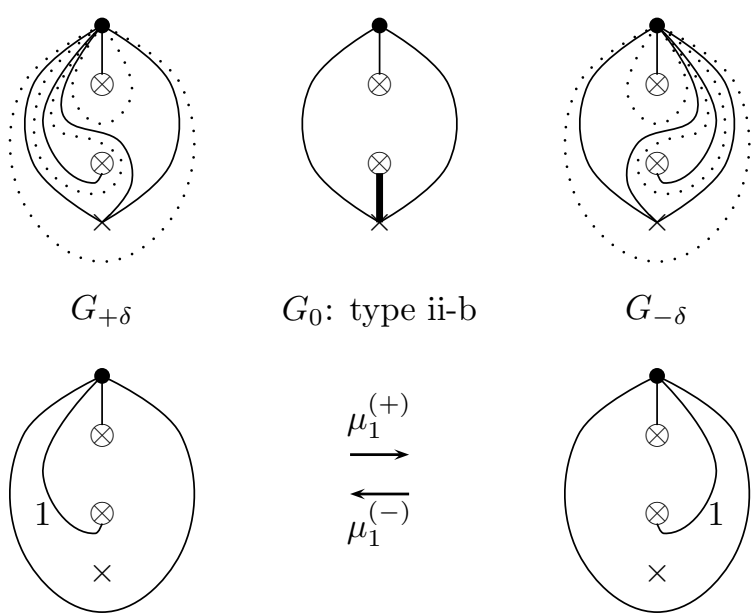

$T_{+\delta}:$ type ii

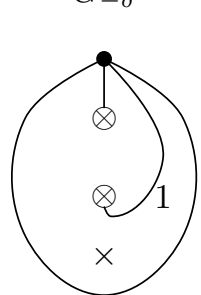

$T_{-\delta}$ : type ii

Figure 19. Mutations of Stokes graphs and signed flips of Stokes triangulations. 
Stokes triangulations associated with $G_{ \pm \delta}$ defined in the previous subsection. Note that the zeros and poles are common for $G_{ \pm \delta}$; therefore, marked points, orbifold points, and midpoints, respectively, in $T_{-\delta}$ and $T_{+\delta}$ are naturally identified.

The following property is the conclusion of this section.

Theorem 3.17. If $G(\phi)$ contains a type $i$ (resp. type ii) configuration, each of the Stokes triangulation $T_{+\delta}$ and $T_{-\delta}$ contains a type $i$ (resp. type ii) piece such that $T_{ \pm \delta}$ undergo signed flips $T_{-\delta}=\mu_{k}^{(+)}\left(T_{+\delta}\right)$ and $T_{+\delta}=\mu_{k}^{(-)}\left(T_{-\delta}\right)$ at the pending arcs therein under the natural identification of the labels of $T_{+\delta}$ and $T_{-\delta}$.

In Figure 19 we show explicitly how this happens in three essential cases of type i-a, ii-a, and ii-b. The rest of the cases are similar.

\section{Mutation Formula of Voros Symbols FOR Signed Flips}

4.1. Simple paths and simple cycles. In this subsection we extend the notion of the simple paths and the simple cycles of a saddle-free Stokes graph defined in IN14 to the simple pole case.

Let $\phi$ be the quadratic differential associated with the Schrödinger equation (2.1). Recall that we call elements of $H_{1}\left(\hat{\Sigma} \backslash\left(\hat{P}_{0} \cup \hat{P}_{\mathrm{s}}\right), \hat{P}_{\infty}\right)$ and $H_{1}(\hat{\Sigma} \backslash \hat{P})$ paths and cycles, respectively (see Section 2.6). Let $\beta^{*}$ (resp., $\gamma^{*}$ ) be the image of a path $\beta$ (resp., a cycle $\gamma$ ) by the covering involution of $\hat{\Sigma}$ while keeping the direction. Let

$$
\begin{aligned}
\operatorname{Sym}\left(H_{1}\left(\hat{\Sigma} \backslash\left(\hat{P}_{0} \cup \hat{P}_{\mathrm{s}}\right), \hat{P}_{\infty}\right)\right) & =\left\{\beta \in H_{1}\left(\hat{\Sigma} \backslash\left(\hat{P}_{0} \cup \hat{P}_{\mathrm{s}}\right), \hat{P}_{\infty}\right) \mid \beta^{*}=\beta\right\}, \\
\operatorname{Sym}\left(H_{1}(\hat{\Sigma} \backslash \hat{P})\right) & =\left\{\gamma \in H_{1}(\hat{\Sigma} \backslash \hat{P}) \mid \gamma^{*}=\gamma\right\} .
\end{aligned}
$$

We introduce the quotients of the homology groups,

$$
\begin{aligned}
\Gamma^{\vee} & =H_{1}\left(\hat{\Sigma} \backslash\left(\hat{P}_{0} \cup \hat{P}_{\mathrm{s}}\right), \hat{P}_{\infty}\right) / \operatorname{Sym}\left(H_{1}\left(\hat{\Sigma} \backslash\left(\hat{P}_{0} \cup \hat{P}_{\mathrm{s}}\right), \hat{P}_{\infty}\right)\right), \\
\Gamma & =H_{1}(\hat{\Sigma} \backslash \hat{P}) / \operatorname{Sym}\left(H_{1}(\hat{\Sigma} \backslash \hat{P})\right) .
\end{aligned}
$$

Remark 4.1. By definition, the formal series $S_{\text {odd }}(z, \eta)$ is anti-invariant under the covering involution $*$ of $\hat{\Sigma}: S_{\text {odd }}\left(z^{*}, \eta\right)=-S_{\text {odd }}(z, \eta)$. Hence,

$$
\int_{\beta}\left(S_{\text {odd }}(z, \eta)-\eta \sqrt{Q_{0}(z)}\right) d z=0, \quad \oint_{\gamma} S_{\text {odd }}(z, \eta) d z=0
$$

hold for any $\beta \in \operatorname{Sym}\left(H_{1}\left(\hat{\Sigma} \backslash\left(\hat{P}_{0} \cup \hat{P}_{\mathrm{S}}\right), \hat{P}_{\infty}\right)\right)$ and any $\gamma \in \operatorname{Sym}\left(H_{1}(\hat{\Sigma} \backslash \hat{P})\right)$. Therefore, Voros symbols for $\beta \in \Gamma^{\vee}$ and $\gamma \in \Gamma$ are well-defined.

In this subsection, we assume

- the Stokes graph $G(\phi)$ of (2.1) is saddle-free.

Then, we can assign the simple path $\beta_{i} \in \Gamma^{\vee}$ and the simple cycle $\gamma_{i} \in \Gamma$ to each Stokes region $D_{i}(i=1, \ldots, n)$ which is not a half plane, as follows. If $D_{i}$ is a regular or degenerate horizontal strip, they are defined by Figures 20 and 21 as in [IN14, Section 6]. Here the signs $\oplus$ and $\ominus$ in the figures are assigned by the same rule as in Section 2.8. If $D_{i}$ is a horizontal strip of simple-pole type, they are newly defined by Figure 22. Note that the intersection form (2.34) is well-defined on the subgroups of $\Gamma^{\vee}$ and $\Gamma$ generated by the simple paths and simple cycles, respectively.

Let $T=T(\phi)=\left(\alpha_{i}\right)_{i=1}^{n}$ be the labeled Stokes triangulation associated with $G(\phi)$ defined in Section 3.4, and let $B=B(T)$ be the signed adjacency matrix of $T$. Let 


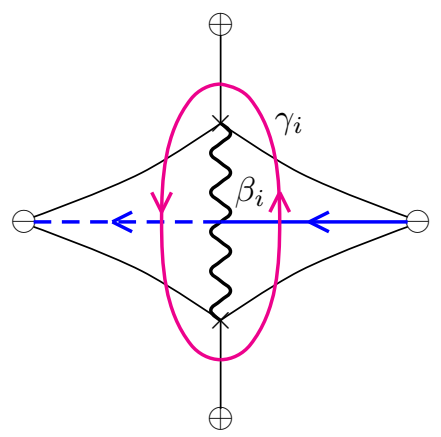

Figure 20. Simple path and simple cycle for a regular horizontal strip $D_{i}$ not surrounding a degenerate horizontal strip.
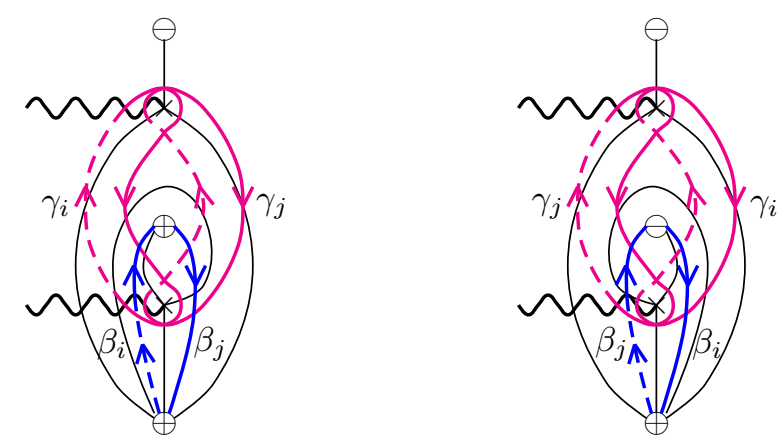

Figure 21. Simple paths and simple cycles for a degenerate horizontal strip $D_{i}$ and for the regular horizontal strip $D_{j}$ surrounding $D_{i}$.

$d_{i}(i=1, \ldots, n)$ be the weight of the arc $\alpha_{i}$. Recall that $\tilde{B}=\left(\tilde{b}_{i j}\right)_{i, j=1}^{n}=D^{-1} B$ is a skew-symmetric integer matrix (see (3.4) and (3.5)).

Proposition 4.2. The following properties hold.

(a). Duality.

$$
\left\langle\gamma_{i}, \beta_{j}\right\rangle=\delta_{i j}
$$

(b). Intersection formula.

$$
\left(\gamma_{i}, \gamma_{j}\right)=\tilde{b}_{i j}
$$

(c). Decomposition formula. As an element of $\Gamma^{\vee}, \gamma_{i}$ decomposes as follows.

$$
\gamma_{i}=\sum_{j=1}^{n} \tilde{b}_{j i} \beta_{j} .
$$

Proof. They are known in the non-simple pole case in [IN14, Propositions 6.25$6.27]$. Thus, it is enough to prove them only when horizontal strips of simple-pole type are involved. This is done by inspecting Figures 20,22 


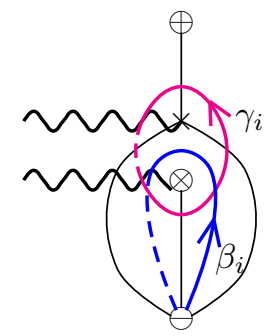

FiguRE 22. Simple path and simple cycle for a horizontal strip $D_{i}$ of simple-pole type.

4.2. Mutation formula of the simple paths and the simple cycles. Next let us give the mutation formula of the simple paths and the simple cycles for signed flips.

We consider the situation of Section 2.7 (and Theorem 3.17). Let $Q^{(\theta)}(z, \eta)$ be the $S^{1}$-family of the potentials of the Schrödinger equation defined in (2.27). In this subsection we assume that

- the Stokes graph $G_{0}=G(\phi)$ of (2.1) (which corresponds to $\theta=0$ ) has a unique Stokes segment $\ell_{0}$, which is of type II.

Fix a $\operatorname{sign} \varepsilon= \pm$. As is explained in Section 2.7 for a sufficiently small $\delta>$ 0 , the Stokes graphs $G=G\left(e^{2 i \varepsilon \delta} \phi\right)$ and $G^{\prime}=G\left(e^{-2 i \varepsilon \delta} \phi\right)$ for $Q^{(+2 i \varepsilon \delta)}(z, \eta)$ and $Q^{(-2 i \varepsilon \delta)}(z, \eta)$, respectively, are saddle-free. Therefore, to the Stokes graph $G$ (resp., $G^{\prime}$ ) and labels $i=1, \ldots, n$ assigned to its Stokes regions, we can associate the labeled Stokes triangulation $T=T\left(e^{2 i \varepsilon \delta} \phi\right)$ (resp., $T^{\prime}=T\left(e^{-2 i \varepsilon \delta} \phi\right)$ ), the simple paths $\beta_{1}, \ldots, \beta_{n} \in \Gamma^{\vee}$ (resp., $\beta_{1}^{\prime}, \ldots, \beta_{n}^{\prime} \in \Gamma^{\vee}$ ), and the simple cycles $\gamma_{1}, \ldots, \gamma_{n} \in \Gamma$ (resp., $\gamma_{1}^{\prime}, \ldots, \gamma_{n}^{\prime} \in \Gamma$ ). Here we have used the same identification of paths and cycles on the Riemann surfaces $\hat{\Sigma}$ of $e^{+i \varepsilon \delta} \sqrt{\phi}$ and $\hat{\Sigma}^{\prime}$ for $e^{-i \varepsilon \delta} \sqrt{\phi}$ explained in Section 2.7. We assign the labels by the same way as in Theorem 3.17 these labeled Stokes triangulations are related by the signed mutation $T^{\prime}=\mu_{k}^{(\varepsilon)}(T)$ with $k$ being the label of the pending $\operatorname{arc}$ in $T$ to be flipped.

Proposition 4.3. Mutation formula. Let $B=B(T)$. Then, the following relations hold.

$$
\begin{aligned}
& \beta_{i}^{\prime}= \begin{cases}-\beta_{k}+d_{k} \sum_{j=1}^{n}\left[-\varepsilon \tilde{b}_{j k}\right]_{+} \beta_{j} & i=k \\
\beta_{i} & i \neq k,\end{cases} \\
& \gamma_{i}^{\prime}= \begin{cases}-\gamma_{k} & i=k \\
\gamma_{i}+d_{k}\left[\varepsilon \tilde{b}_{k i}\right]_{+} \gamma_{k} & i \neq k .\end{cases}
\end{aligned}
$$

Proof. Again, they are known in the non-simple pole case in IN14, Propositions 6.28]. Thus, it is enough to prove them only when horizontal strips of simple-pole type are involved. Furthermore, it is enough to prove only (4.10) thanks to the duality (4.6). This is done by inspecting Figures 19222 and also [N14, Figures $36]$. 

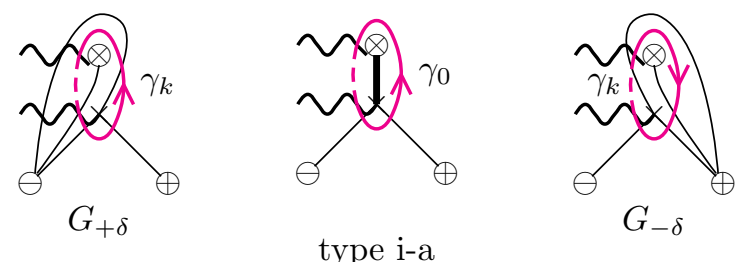

FiguRE 23. Relation between $\gamma_{0}$ and $\gamma_{k}$.

4.3. Mutation formula of Voros symbols for signed flips. Here we reformulate Theorem 2.17 in view of cluster algebra theory. We follow the same argument for the non-simple pole case treated in [IN14, Section 7].

Below we consider the same situation as the previous subsection: Fix a sign $\varepsilon= \pm$, and consider a mutation of Stokes graphs (for a reduction of the Stokes segment $\ell_{0}$ which is of type II) which induces the signed flip $\mu_{k}^{(\varepsilon)}$ to the associated labeled Stokes triangulations.

Let $G, G^{\prime}, T, T^{\prime}, \beta_{i}, \beta_{i}^{\prime} \in \Gamma^{\vee}(i=1, \ldots, n)$ and $\gamma_{i}, \gamma_{i}^{\prime} \in \Gamma(i=1, \ldots, n)$ be the same ones defined in the previous subsection. Let $\gamma_{0}$ be the cycle surrounding the Stokes segment $\ell_{0}$ as indicated in Figure 7 whose orientation is determined by the condition (2.33).

Lemma 4.4. The equality $\gamma_{0}=\varepsilon \gamma_{k}$ holds.

Proof. This is checked by inspecting Figure 23, where we assume that $\ell_{0}$ is in the type i-a configuration in Figure 18, The other cases can be checked similarly by using Figure 19,

Let $e^{W_{\beta}^{(\theta)}}=e^{W_{\beta}^{(\theta)}(\eta)}$ and $e^{V_{\gamma}^{(\theta)}}=e^{V_{\gamma}^{(\theta)}(\eta)}$ be the Voros symbols for the Schrödinger equation with the potential $Q^{(\theta)}(z, \eta)$, respectively. We also set

$$
\mathcal{S}_{ \pm \varepsilon}\left[e^{W_{\beta}}\right](\eta)=\lim _{\delta \rightarrow+0} \mathcal{S}\left[e^{W_{\beta}^{( \pm \varepsilon \delta)}}\right](\eta), \quad \mathcal{S}_{ \pm \varepsilon}\left[e^{V_{\gamma}}\right](\eta)=\lim _{\delta \rightarrow+0} \mathcal{S}\left[e^{V_{\gamma}^{( \pm \varepsilon \delta)}}\right](\eta)
$$

First, we present the following intermediate result obtained from Theorem 2.17

Proposition 4.5. For any path $\beta \in \Gamma^{\vee}$ and any cycle $\gamma \in \Gamma$, we have

$$
\begin{aligned}
& \mathcal{S}_{-\varepsilon}\left[e^{W_{\beta}}\right]=\mathcal{S}_{+\varepsilon}\left[e^{W_{\beta}}\right]\left(1+\left(t_{k}+t_{k}^{-1}\right)\left(\mathcal{S}_{+\varepsilon}\left[e^{V_{\gamma_{k}}}\right]\right)^{\varepsilon}+\left(\mathcal{S}_{+\varepsilon}\left[e^{2 V_{\gamma_{k}}}\right]\right)^{\varepsilon}\right)^{-\left\langle\gamma_{k}, \beta\right\rangle} \\
& \mathcal{S}_{-\varepsilon}\left[e^{V_{\gamma}}\right]=\mathcal{S}_{+\varepsilon}\left[e^{V_{\gamma}}\right]\left(1+\left(t_{k}+t_{k}^{-1}\right)\left(\mathcal{S}_{+\varepsilon}\left[e^{V_{\gamma_{k}}}\right]\right)^{\varepsilon}+\left(\mathcal{S}_{+\varepsilon}\left[e^{2 V_{\gamma_{k}}}\right]\right)^{\varepsilon}\right)^{-\left(\gamma_{k}, \gamma\right)}
\end{aligned}
$$

where $t_{k}=\exp \left(\pi i \sqrt{1+4 b_{k}}\right), b_{k}=\lim _{z \rightarrow s_{k}}\left(\left(z-s_{k}\right)^{2} Q_{2}(z)\right)$ with $s_{k}$ being the simple pole attached to $\ell_{0}$.

Proof. They follow from Theorem 2.17, by using Lemma 4.4 and the equality (2.56). See the proof of [N14, Proposition 7.3] for more detail.

Let us introduce

$$
\begin{gathered}
e^{W_{i}}=e^{W_{\beta_{i}}^{(\varepsilon \delta)}}, \quad e^{V_{i}}=e^{V_{\gamma_{i}}^{(\varepsilon \delta)}}, \quad e^{v_{i}}=e^{\eta v_{\gamma_{i}}^{(\varepsilon \delta)},} \\
e^{W_{i}^{\prime}}=e^{W_{\beta_{i}^{\prime}}^{(-\varepsilon \delta)}}, \quad e^{V_{i}^{\prime}}=e^{V_{\gamma_{i}^{\prime}}^{(-\varepsilon \delta)}}, \quad e^{v_{i}^{\prime}}=e^{\eta v_{\gamma_{i}^{\prime}}^{(-\varepsilon \delta)}} .
\end{gathered}
$$


where

$$
v_{\gamma}^{(\theta)}=e^{i \theta} \int_{\gamma} \sqrt{Q_{0}(z)} d z
$$

By (4.8) we have

$$
e^{V_{i}}=e^{v_{i}} \prod_{j=1}^{n}\left(e^{W_{j}}\right)^{\tilde{b}_{j i}}, \quad e^{V_{i}^{\prime}}=e^{v_{i}^{\prime}} \prod_{j=1}^{n}\left(e^{W_{j}^{\prime}}\right)^{\tilde{b}_{j i}^{\prime}},
$$

where $\tilde{B}=D^{-1} B$ and $\tilde{B}^{\prime}=D^{-1} B^{\prime}$ with $B$ and $B^{\prime}$ being the signed adjacency matrices of $T$ and $T^{\prime}$, respectively.

Now we present the second main result of the paper.

Theorem 4.6. We set

$$
\begin{array}{lll}
y_{i}=\lim _{\delta \rightarrow+0} e^{v_{i}}, & x_{i}=\lim _{\delta \rightarrow+0} \mathcal{S}\left[e^{W_{i}}\right], & \hat{y}_{i}=\lim _{\delta \rightarrow+0} \mathcal{S}\left[e^{V_{i}}\right], \\
y_{i}^{\prime}=\lim _{\delta \rightarrow+0} e^{v_{i}^{\prime}}, & x_{i}^{\prime}=\lim _{\delta \rightarrow+0} \mathcal{S}\left[e^{W_{i}^{\prime}}\right], & \hat{y}_{i}^{\prime}=\lim _{\delta \rightarrow+0} \mathcal{S}\left[e^{V_{i}^{\prime}}\right] .
\end{array}
$$

Then, the following relations hold:

$$
\begin{aligned}
& y_{i}^{\prime}= \begin{cases}y_{k}^{-1} & i=k \\
y_{i}\left(y_{k}^{\left[\varepsilon \tilde{b}_{k i}\right]_{+}}\right)^{2} \quad i \neq k,\end{cases} \\
& x_{i}^{\prime}= \begin{cases}x_{k}^{-1}\left(\prod_{j=1}^{n} x_{j}^{\left[-\varepsilon \tilde{b}_{j k}\right]_{+}}\right)^{2}\left(1+\left(t_{k}+t_{k}^{-1}\right) \hat{y}_{k}^{\varepsilon}+\hat{y}_{k}^{2 \varepsilon}\right) & i=k \\
x_{i} & i \neq k,\end{cases} \\
& \hat{y}_{i}^{\prime}= \begin{cases}\hat{y}_{k}^{-1} & i=k \\
\hat{y}_{i}\left(\hat{y}_{k}^{\left[\varepsilon \tilde{b}_{k i}\right]_{+}}\right)^{2}\left(1+\left(t_{k}+t_{k}^{-1}\right) \hat{y}_{k}^{\varepsilon}+\hat{y}_{k}^{2 \varepsilon}\right)^{-\tilde{b}_{k i}} & i \neq k .\end{cases}
\end{aligned}
$$

Proof. The relation (4.20) follows from (4.10). To get the relation (4.21), we specialize (4.12) with $\beta=\beta_{i}^{\prime}$, then use (4.6) and (4.9). To get the relation (4.22), we specialize (4.13) with $\gamma=\gamma_{i}^{\prime}$, then use (4.7) and (4.10).

4.4. Generalized cluster algebras. Let us explain that the relations (4.20)(4.22) are indeed (an enhancement of) the mutation in generalized cluster algebras recently introduced by CS14. Here, for clarity, we minimize the setting of CS14 just to be fitted for our situation.

Let us recall the definition of a seed in cluster algebras. See [FZ07] for detail. We fix a semifield $\mathbb{P}$, which is a multiplicative abelian group with addition $\oplus$. Let $\mathbb{Z P}$ be the group ring of $\mathbb{P}$, and let $\mathbb{Q P}$ be the field of fractions of $\mathbb{Z P}$. Let $\mathbb{Q P}\left(x^{0}\right)$ be the rational function field in the initial $x$-variables $x_{1}^{0}, \ldots, x_{n}^{0}$ over $\mathbb{Q P}$. A seed is a triplet $(B, x, y)$, where $B=\left(b_{i j}\right)_{i, j=1}^{n}$ is a skew-symmetrizable matrix, $x=\left(x_{1}, \ldots, x_{n}\right)$ is an $n$-tuple with $x_{i} \in \mathbb{Q P}\left(x^{0}\right)$, and $y=\left(y_{1}, \ldots, y_{n}\right)$ is an $n$-tuple with $y_{i} \in \mathbb{P}$.

To consider the generalized cluster algebras limited to our case, it is crucial to assume that the matrix $B$ factorizes as $B=D \tilde{B}$, where $D=\left(d_{i} \delta_{i j}\right)$ is a diagonal matrix with $d_{i} \in\{1,2\}$ and $\tilde{B}$ is an integer matrix, which is indeed true in our situation (see (3.4) and (3.5)). (In general, $\tilde{B}$ is not necessarily skew-symmetric.) Let $I_{2}:=\left\{i \in\{1, \ldots, n\} \mid d_{i}=2\right\}$, and let us fix $z_{i} \in \mathbb{P}$ for each $i \in I_{2}$ arbitrarily. 
We consider the following "mutation" $\left(B^{\prime}, x^{\prime}, y^{\prime}\right)=\mu_{k}(B, x, y)$ of seeds at $k=$ $1, \ldots, n: B^{\prime}=\mu_{k}(B)$ is the usual one in (3.6), and

$$
\begin{aligned}
& y_{i}^{\prime}= \begin{cases}y_{k}^{-1} & i=k \\
y_{i} y_{k}^{\left[\varepsilon \tilde{b}_{k i}\right]_{+}}\left(1 \oplus y_{k}^{\varepsilon}\right)^{-\tilde{b}_{k i}} & i \neq k, d_{k}=1 \\
y_{i}\left(y_{k}^{\left[\varepsilon \tilde{b}_{k i}\right]_{+}}\right)^{2}\left(1 \oplus z_{k} y_{k}^{\varepsilon} \oplus y_{k}^{2 \varepsilon}\right)^{-\tilde{b}_{k i}} & i \neq k, d_{k}=2,\end{cases} \\
& x_{i}^{\prime}= \begin{cases}x_{k}^{-1}\left(\prod_{j=1}^{n} x_{j}^{\left[-\varepsilon \tilde{b}_{j k}\right]_{+}}\right) \frac{1+\hat{y}_{k}^{\varepsilon}}{1 \oplus y_{k}^{\varepsilon}} & i=k, d_{k}=1 \\
x_{k}^{-1}\left(\prod_{j=1}^{n} x_{j}^{\left[-\varepsilon \tilde{b}_{j k}\right]_{+}}\right)^{2} \frac{1+z_{k} \hat{y}_{k}^{\varepsilon}+\hat{y}_{k}^{2 \varepsilon}}{1 \oplus z_{k} y_{k}^{\varepsilon} \oplus y_{k}^{2 \varepsilon}} & i=k, d_{k}=2 \\
x_{i} & i \neq k,\end{cases}
\end{aligned}
$$

where $\varepsilon= \pm$ and we set

$$
\hat{y}_{i}=y_{i} \prod_{j=1}^{n} x_{j}^{\tilde{b}_{j i}}
$$

We note that $B^{\prime}$ again factorizes as $B^{\prime}=D \tilde{B}^{\prime}$ with the same $D$. The relations involving $k$ with $d_{k}=2$ in (4.23) and (4.24) are unusual in ordinary cluster algebras, but they are indeed an example of the mutation for generalized cluster algebras defined in CS14. It is easy to see that the above expressions do not depend on the choice of the $\operatorname{sign} \varepsilon$. Furthermore, $\hat{y}$-variables in (4.25) transform as $y$-variables, namely,

$$
\hat{y}_{i}^{\prime}= \begin{cases}\hat{y}_{k}^{-1} & i=k \\ \hat{y}_{i} \hat{y}_{k}^{\left[\varepsilon \tilde{b}_{k i}\right]_{+}}\left(1+\hat{y}_{k}^{\varepsilon}\right)^{-\tilde{b}_{k i}} & i \neq k, d_{k}=1 \\ \hat{y}_{i}\left(\hat{y}_{k}^{\left[\varepsilon \tilde{b}_{k i}\right]_{+}}\right)^{2}\left(1+z_{k} \hat{y}_{k}^{\varepsilon}+\hat{y}_{k}^{2 \varepsilon}\right)^{-\tilde{b}_{k i}} & i \neq k, d_{k}=2 .\end{cases}
$$

The relations (4.23)-(4.26) with $d_{k}=2$ are already very close to (4.20)-(4.22) and (4.17) under the identification $z_{k}=t_{k}+t_{k}^{-1}$. In fact, the only difference between them is the absence of the factors $1 \oplus y_{k}$ and $1 \oplus z_{k} y_{k}^{\varepsilon} \oplus y_{k}^{2 \varepsilon}$ in (4.20) and (4.21).

Having this discrepancy in mind, we now specialize the coefficient semifield $\mathbb{P}$ to the tropical semifield $\operatorname{Trop}\left(y^{0}, z\right)$, which is the multiplicative free abelian group generated by the initial $y$-variables $y_{1}^{0}, \ldots, y_{n}^{0}$ and $z_{i}\left(i \in I_{2}\right)$ with the tropical sum

$$
\begin{aligned}
& \left(\prod_{i=1}^{n}\left(y_{i}^{0}\right)^{a_{i}}\right)\left(\prod_{i \in I_{2}} z_{i}^{a_{i}^{\prime}}\right) \oplus\left(\prod_{i=1}^{n}\left(y_{i}^{0}\right)^{b_{i}}\right)\left(\prod_{i \in I_{2}} z_{i}^{b_{i}^{\prime}}\right) \\
= & \left(\prod_{i=1}^{n}\left(y_{i}^{0}\right)^{\min \left(a_{i}, b_{i}\right)}\right)\left(\prod_{i \in I_{2}} z_{i}^{\min \left(a_{i}^{\prime}, b_{i}^{\prime}\right)}\right) .
\end{aligned}
$$

As a rule, we start mutation from the initial seed $\left(B^{0}, x^{0}, y^{0}\right)$, where $B^{0}$ is any skew-symmetrizable matrix which factorizes as $B^{0}=D \tilde{B}^{0}$, and $x^{0}$ and $y^{0}$ are the initial $x$ - and $y$-variables; then, repeat mutations. Our first observation is that $z_{k}$ in the factor $1 \oplus z_{k} y_{k}^{\varepsilon} \oplus y_{k}^{2 \varepsilon}$ in (4.24) is superficial, and it never enters in any $y$-variable $y_{i}$ after any sequence of mutations. Thus, the factor $1 \oplus z_{k} y_{k}^{\varepsilon} \oplus y_{k}^{2 \varepsilon}$ in (4.23) and (4.24) are replaced with the factor $1 \oplus y_{k}^{\varepsilon} \oplus y_{k}^{2 \varepsilon}$ without changing the mutation. 
We say that $y_{i}=\prod_{j=1}^{n}\left(y_{j}^{0}\right)^{c_{j}} \in \operatorname{Trop}(y, z)$ is sign-coherent if $\left(c_{j}\right)_{j=1}^{n}$ is nonzero and either $c_{j} \geq 0$ for any $j$ or $c_{j} \leq 0$ for any $j$. In this case we set the tropical sign $\varepsilon\left(y_{i}\right)$ of $y_{i}$ as + or - , according to the sign of $\left(c_{j}\right)_{j=1}^{n}$. Our second observation, which is a familiar idea for ordinary cluster algebras, is that if $y_{k}$ is sign-coherent and if we set $\varepsilon=\varepsilon\left(y_{k}\right)$, then we have $1 \oplus y_{k}^{\varepsilon} \oplus y_{k}^{2 \varepsilon}=1$.

Let us introduce the signed mutation $\left(B^{\prime}, x^{\prime}, y^{\prime}\right)=\mu_{k}^{(\varepsilon)}(B, x, y)$ of seeds $(k=$ $1, \ldots, n ; \varepsilon= \pm)$ for our generalized cluster algebras by replacing (4.23) and (4.24) with

$$
\begin{aligned}
& y_{i}^{\prime}= \begin{cases}y_{k}^{-1} & i=k \\
y_{i}\left(y_{k}^{\left[\varepsilon \tilde{b}_{k i}\right]_{+}}\right)^{d_{k}} & i \neq k,\end{cases} \\
& x_{i}^{\prime}= \begin{cases}x_{k}^{-1}\left(\prod_{j=1}^{n} x_{j}^{\left[-\varepsilon \tilde{b}_{j k}\right]_{+}}\right)\left(1+\hat{y}_{k}^{\varepsilon}\right) & i=k, d_{k}=1 \\
x_{k}^{-1}\left(\prod_{j=1}^{n} x_{j}^{\left[-\varepsilon \tilde{b}_{j k}\right]_{+}}\right)^{2}\left(1+z_{k} \hat{y}_{k}^{\varepsilon}+\hat{y}_{k}^{\varepsilon \varepsilon}\right) & i=k, d_{k}=2 \\
x_{i} & i \neq k,\end{cases}
\end{aligned}
$$

which completely agree with (4.20) and (4.21). We note that the relation (4.26) still holds. This generalizes the signed mutations for ordinary cluster algebras introduced in [IN14, Section 4].

Now we have reached to the conclusion that the signed mutation $\mu_{k}^{(\varepsilon)}$, which appears in Theorem 4.6. coincides with the ordinary mutation $\mu_{k}$ in a generalized cluster algebra if $y_{k}$ is sign-coherent and the $\operatorname{sign} \varepsilon$ is chosen to be the tropical sign of $y_{k}$; otherwise, it is an extension of the ordinary mutation therein.

Remark 4.7. In this paper we concentrated on the signed flips of pending arcs for simplicity. As for the signed flips of ordinary arcs, it was already shown in IN14, Theorem 7.5] that the Voros symbols mutate by (4.28), (4.29), and (4.26) with $d_{k}=1$ in the absence of simple poles. Using Propositions 4.2 and 4.3, we can easily extend the proof therein to show that this is true even in the presence of simple poles. Therefore, the formulas (4.28) and (4.29) integrate the mutation formula of the Voros symbols for all cases of signed flips.

Finally, let us present a conjecture clarifying the role of Stokes triangulations from the cluster algebraic point of view. Let $T$ be a labeled Stokes triangulation of an orbifold $(\mathbf{O}, \mathbf{A})$. Let us consider a sequence of signed flips $T^{\prime}=\mu_{k_{N}}^{\left(\varepsilon_{N}\right)} \circ \cdots \circ$ $\mu_{k_{1}}^{\left(\varepsilon_{1}\right)}(T)$. Let $(B, x, y)$ be a seed with $B=B(T)$, and apply the same sequence of signed mutations to $(B, x, y)$ as $\left(B^{\prime}, x^{\prime}, y^{\prime}\right)=\mu_{k_{N}}^{\left(\varepsilon_{N}\right)} \circ \cdots \circ \mu_{k_{1}}^{\left(\varepsilon_{1}\right)}(B, x, y)$.

Conjecture 4.8. We have $T=T^{\prime}$ if and only if $\left(B^{\prime}, x^{\prime}, y^{\prime}\right)=(B, x, y)$.

This is an extension of [IN14, Conjecture 6.6 (i)].

\section{REFERENCES}

[AIT] T. Aoki, K. Iwaki and T. Takahashi, Exact WKB analysis of Schrödinger equations with a Stokes curve of loop type, in preparation.

[AKT91] T. Aoki, T. Kawai and Y. Takei, The Bender-Wu analysis and the Voros theory, ICM-90 Satellite Conf. Proc. "Special Functions", Springer-Verlag, 1991, pp. 1-29. 
[AKT09] - The Bender-Wu analysis and the Voros theory II, Adv. Stud. in Pure Math., 54, Math. Soc. Japan, 2009, pp.19-94.

[BS13] T. Bridgeland and I. Smith, Quadratic differentials as stability conditions, 2013, arXiv:1302.7030 [math.AG].

[CS14] L. Chekhov and M. Shapiro, Teichmüller spaces of Riemann surfaces with orbifold points of arbitrary order and cluster variables, Int. Math. Res. Notices 2014 (2014), 2746-2772; arXiv:1111.3963 [math-ph].

[Cos09] O. Costin, Asymptotics and Borel Summability, Monographs and Surveys in Pure and Applied Mathematics 141, Chapmann \& Hall/CRC, New York, 2009.

[DDP93] E. Delabaere, H. Dillinger and F. Pham, Résurgence de Voros et périodes des courbes hyperelliptiques, Ann. Inst. Fourier (Grenoble) 43 (1993), 163-199.

[DP99] E. Delabaere and F. Pham, Resurgent methods in semi-classical asymptotics, Ann. Inst. Henri Poincaré 71 (1999), 1-94.

[FST11] A. Felikson, M. Shapiro, and P. Tumarkin, Cluster algebras and triangulated orbifolds, Adv. in Math. 231 (2012), 2953-3002; arXiv:1111.3449 [math.CO].

[FG06] V.V. Fock and A.B. Goncharov, Moduli spaces of local systems and higher Teichmüller theory, Publ. Math. IHES 103 (2006), 1-211; arXiv:math/0311149 [math.AG].

[FST08] S. Fomin, M. Shapiro, and D. Thurston, Cluster algebras and triangulated surfaces. Part I: Cluster complexes, Acta Math. 201 (2008), 83-146; arXiv:math/0608367 [math.RA].

[FT12] S. Fomin and D. Thurston, Cluster algebras and triangulated surfaces. Part II: Lambda lengths, 2012, arXiv:1210.5569 [math.GT].

[FZ03] S. Fomin and A. Zelevinsky, Cluster algebras II. Finite type classification, Invent. Math. 154 (2003), 63-121; arXiv:math/0208229 [math.RA].

[FZ07] , Cluster algebras IV. Coefficients, Compositio Mathematica 143 (2007), 112-164; arXiv:math/0602259 [math.RT].

[GMN13] D. Gaiotto, G.W. Moore, and A. Neitzke, Wall-crossing, Hitchin systems, and the WKB approximation, Adv. in Math. 234 (2013), 239-403; arXiv:0907.3987 [hepth].

[GSV05] M. Gekhtman, M. Shapiro, and A. Vainshtein, Cluster algebras and WeilPetersson forms, Duke Math. J. 127 (2005), 291-311; arXiv:math/0309138 [math.QA].

[IN14] K. Iwaki and T. Nakanishi, Exact WKB analysis and cluster algebras, 2014, arXiv:1401.7094 [math CA], to appear in J. Phys. A.

[KKKT10] S. Kamimoto, T. Kawai, T. Koike and Y. Takei, On the WKB-theoretic structure of a Schrödinger operator with a merging pair of a simple pole and a simple turning point, Kyoto J. Math. 50 (2010), 101-164.

[KK11] S. Kamimoto and T. Koike, On the Borel summability of WKB-theoretic transformation series, preprint of RIMS-1726.

[KT05] T. Kawai and Y. Takei, Algebraic Analysis of Singular Perturbation Theory, Translations of Mathematical Monographs, volume 227, American Mathematical Society, 2005.

[Koi00] T. Koike, On the exact WKB analysis of second order linear ordinary differential equations with simple poles, Publ. RIMS, Kyoto Univ. 36 (2000), 297-319.

[KS] T. Koike and R. Schäfke, On the Borel summability of WKB solutions of Schrödinger equations with polynomial potentials and its application, in preparation; also Talk given by T. Koike in the RIMS workshop "Exact WKB analysis - Borel summability of WKB solutions", September, 2010.

[KT11] T. Koike and Y. Takei, On the Voros Coefficient for the Whittaker Equation with a Large Parameter - Some Progress around Sato's Conjecture in Exact WKB Analysis, Publ. RIMS, Kyoto Univ. 47 (2011), 375-395. 
[Qiu14] Y. Qiu, On the spherical twists on 3-Calabi-Yau categories from marked surfaces, 2014, arXiv:1407.0806.

[Str84] K. Strebel, Quadratic Differentials, Springer-Verlag, 1984.

[Vor83] A. Voros, The return of the quartic oscillator. The complex WKB method, Ann. Inst. Henri Poincaré 39(1983), 211-338.

Research Institute for Mathematical Sciences, Kyoto University, Kyoto, 657-8501, JAPAN

E-mail address: iwaki@kurims.kyoto-u.ac.jp

Graduate School of Mathematics, Nagoya University, Chikusa-Ku, Nagoya, 464-8604, JAPAN

E-mail address: nakanisi@math.nagoya-u.ac.jp 